University of Nebraska - Lincoln

DigitalCommons@University of Nebraska - Lincoln

September 1996

\title{
Fundamental Aspects of Atomic Photoionization with High- Brightness Light Sources
}

Anthony F. Starace

University of Nebraska-Lincoln, astarace1@unl.edu

Steven T. Manson

Georgia State University

Follow this and additional works at: https://digitalcommons.unl.edu/physicsstarace

Part of the Physics Commons

Starace, Anthony F. and Manson, Steven T., "Fundamental Aspects of Atomic Photoionization with HighBrightness Light Sources" (1996). Anthony F. Starace Publications. 154.

https://digitalcommons.unl.edu/physicsstarace/154

This Article is brought to you for free and open access by the Research Papers in Physics and Astronomy at DigitalCommons@University of Nebraska - Lincoln. It has been accepted for inclusion in Anthony F. Starace Publications by an authorized administrator of DigitalCommons@University of Nebraska - Lincoln. 


\title{
Fundamental Aspects of Atomic Photoionization with High-Brightness Light Sources
}

\author{
Anthony F. Starace \\ Department of Physics and Astronomy, \\ University of Nebraska-Lincoln, Lincoln, Nebraska 68588-0111 \\ Steven T. Manson \\ Department of Physics and Astronomy, \\ Georgia State University, Atlanta, Georgia 30303-3083
}

\section{Introduction}

The construction of numerous new synchrotron light sources worldwide is providing the means to study atomic and molecular photoionization processes at a level of detail that is unparalleled in even the recent past. Correspondingly, in theory, the availability of supercomputers and, perhaps more important, relatively inexpensive computer workstations, has permitted theoretical calculations to tackle more complex processes than ever before. In short, these technical developments are creating an unprecedented amount of data to be analyzed and understood.

In this volume, many of the experimental and theoretical advances of recent years will be described. We take the point of view in this introductory paper that while agreement of theory and experiment is always desirable, the main goal of researchers in this field should be to advance our understanding of the key physics governing photoionization processes in as simple a way as possible. As there can be no general prescription for achieving such a goal, we therefore present an eclectic set of examples of recent advances in experimental techniques or theoretical analysis which have achieved such simplicity in the face of complexity. For clarity, however, we review briefly beforehand some essentials of the theory of photoionization.

\section{Brief Review of Theory}

In the dipole approximation, which is excellent for low-energy photoionization, the cross section for a photoionizing transition induced by an unpolarized beam of photons of energy $h v$ from an initial state $|i\rangle$ to a final state $|f\rangle$ given by [1]

$$
\sigma_{\text {if }}=\frac{4 \pi^{2} \alpha a_{0}^{2}}{3 g_{\mathrm{i}}}(h v)\left|M_{\mathrm{if}}\right|^{2}
$$

where $\alpha$ is the fine structure constant, $a_{0}$ is the Bohr radius, and $g_{\mathrm{i}}$ is the statistical weight of the initial state. The absolute square of the matrix element is given by [2] 


$$
\left|M_{\mathrm{if}}\right|^{2}=\sum_{i, f}\left|\left\langle f\left|\sum_{j} \vec{r}_{j}\right| i\right\rangle\right|^{2}
$$

where $\vec{r}_{j}$ is the position coordinate of the $j$ th electron, the sums are over the degenerate initial and final magnetic substates, and the wave functions are normalized such that

$$
\langle i \mid i\rangle=1,\left\langle f \mid f^{\prime}\right\rangle=\delta\left(\varepsilon-\varepsilon^{\prime}\right)
$$

where $\varepsilon$ is the photoelectron energy. Details and examples for a number of cases are given in References 3 through 7.

The photoelectron angular distribution resulting from photoionization of state $|i\rangle$ by linearly polarized photons leaving the ion in state $|j\rangle$ is given by [8-10]

$$
\frac{d \sigma_{\mathrm{ij}}}{d \Omega}=\frac{\sigma_{\mathrm{ij}}}{4 \pi}\left[1+\beta_{\mathrm{ij}} P_{2}(\cos \theta)\right]
$$

where $\sigma_{\mathrm{ij}}$ is the total photoionization cross section for producing state $|j\rangle$ of the ion, $\theta$ is the angle between the photon's polarization vector and the photoelectron's momentum direction, $P_{2}(\mathrm{x})=\left(3 \mathrm{x}^{2}-1\right) / 2$, and $\beta_{\mathrm{ij}}$ is the asymmetry parameter. There are various equivalent ways of obtaining $\beta_{\mathrm{ij}}[11,12]$; the angular momentum transfer formulation [11,12] is presented here since the essential determinants of the angular distribution emerge most clearly in this formulation.

Consider the ejection of a photoelectron from an unpolarized atom $A$,

$$
A\left(J_{0} \pi_{0}\right)+\left(j_{\gamma}=1, \pi_{\gamma}=-1\right) \rightarrow\left(J_{\mathrm{c}} \pi_{\mathrm{c}}\right)+e^{-}\left(l s j, \pi_{\mathrm{e}}=(-1)^{l}\right)
$$

where $\pi$ denotes the parity quantum number of the various constituents of the process. The cross section can be partitioned into incoherent contributions characterized by alternative values of the angular momentum transfer, [13]

$$
\vec{j}_{\mathrm{t}}=\vec{j}_{\gamma}-\vec{l}=\vec{J}_{\mathrm{c}}+\vec{s}-\vec{J}_{0}
$$

The possible values of $j_{\mathrm{t}}$ are determined from equation (6), subject to the constraints of angular momentum and parity conservation. The allowed values of $j_{\mathrm{t}}$ are characterized by their parity according to $\pi_{0} \pi_{c}= \pm(-1)^{j_{t}}$, where values of $j_{t}$ for which the plus sign is required are called "parity-favored" and those requiring the minus sign are called "parity-unfavored." The key point here is that for parity-unfavored values of $j_{\mathrm{t}^{\prime}} \beta\left(j_{\mathrm{t}}\right)=-1$, independent of energy; for parity-favored values of $j_{t^{\prime}} \beta\left(j_{t}\right)$ is, in general, energy- dependent and varies between the limits of -1 and +2 . The asymmetry parameter for the entire transition is then given by the following weighted average of the various $\beta\left(j_{t}\right)$ parameters:

$$
\begin{gathered}
\beta_{\mathrm{ij}}=\left(\sum_{j_{\mathrm{t}}}^{\mathrm{fav}} \sigma\left(j_{\mathrm{t}}\right)_{\mathrm{fav}} \beta\left(j_{\mathrm{t}}\right)_{\mathrm{fav}}-\sum_{j_{\mathrm{t}}}^{\mathrm{unf}} \sigma\left(j_{\mathrm{t}}\right)_{\mathrm{unf}}\right) / \sigma_{\mathrm{ij}} \\
\sigma_{\mathrm{ij}}=\sum_{j_{\mathrm{t}}} \sigma\left(j_{\mathrm{t}}\right)
\end{gathered}
$$

Detailed expressions for $\sigma\left(j_{\mathrm{t}}\right)$ and $\beta\left(j_{\mathrm{t}}\right)_{\text {fav }}$ are given elsewhere. [6, 11-13]

Of course, in order to actually compute cross sections and angular distributions, wave functions for initial and final states are required. Discussion of these wave functions is beyond the scope of this chapter, but numerous reviews [3-7], along with several chapters of the present volume, deal with this subject extensively. 


\section{Examples of the Essential Physics Underlying Complex Photoionization Phenomena}

In this main section we discuss a number of instances in which rather complex photoionization phenomena have been interpreted simply by novel theoretical or experimental means. We hasten to add that these examples are not intended to be complete. Many of them have involved one or the other of the authors of this chapter.

\subsection{Doubly Excited State Spectra of $\mathrm{H}^{-}$and $\mathrm{He}$}

The helium atom and the negative ion of the hydrogen atom are fundamental three-body Coulomb systems. Their photoionization and photodetachment spectra have attracted much experimental and theoretical interest for decades. Only recently, however, through the use of lasers and of high-brightness synchrotron light, has it been possible to obtain experimental spectra on their doubly excited states converging to thresholds higher than $n=2$. Thus, Harris et al. [14] observed doubly excited spectra of $\mathrm{H}^{-}$converging to the $n=4-8$ thresholds of $\mathrm{H}^{+}$. Similarly, Domke et al. [15] recently reported the high-resolution photoionization study of the doubly excited He states below the $n=2-7$ thresholds of $\mathrm{He}^{+}$. Both of these experimental spectra are very rich, having a wealth of detail. Furthermore, in addition to providing much more detail on doubly excited state spectra than earlier measurements focused only on the $n=2$ threshold [16], so too did these measurements permit theorists to devise a more general interpretation of doubly excited state spectra than provided in earlier work. [17,18]

The pioneering measurements of Madden and Codling [16] on resonances converging to the $\mathrm{He}^{+}(n=2)$ threshold were surprising in two respects. First, an independent electron model picture leads one to expect three ${ }^{1} P^{O}$ Rydberg series of resonances: $\operatorname{He} 2 \sin \left({ }^{1} P^{O}\right), \operatorname{He} 2 p n s\left({ }^{1} P^{O}\right)$, and $\mathrm{He} 2 \mathrm{pnd}\left({ }^{1} \mathrm{P}^{\mathrm{O}}\right)$. However, experiment observed the presence of only two series. Second, of the two series that were observed, one was very prominent and the other was barely observable. Cooper et a1. [17] interpreted the two observed series as different linear combinations of the first two independent particle model series listed above, i.e., $\operatorname{He}(2 s n p \pm 2 p n s){ }^{1} P^{O}$. They postulated that the "+" series had much more intensity than the "-" series as a result of cancellations in the dipole amplitude to the "-" states. Their interpretation, however, did not explain the absence of the $\mathrm{He} 2 p n d\left({ }^{1} \mathrm{P}^{\mathrm{O}}\right)$ series from the experimental observations.

It was Macek's use of an adiabatic hyperspherical representation for these two-electron states that finally explained (at least qualitatively) all observed characteristics of these spectra.[18] In hyperspherical coordinates the six independent electron coordinates $r_{1}, r_{2}, r_{1}$, and $r_{2}$ are replaced by $R, \alpha, r_{1}$, and $r_{2}$, where the hyperradius $R$ defined by

$$
R \equiv\left(r_{1}^{2}+r_{2}^{2}\right)^{1 / 2}
$$

measures the "size" of a two-electron state, and the radial angle $\alpha$, defined by

$$
\alpha \equiv \arctan \left(r_{2} / r_{1}\right)
$$

measures the radial correlation of the two electrons. In particular, $\alpha=0$ or $\pi / 2$ corresponds to independent particle motion in which one electron is near the nucleus and the other is very far away; $\alpha=\pi / 4$ on the other hand corresponds to a doubly excited state in which the two electrons are at equal distances from the nucleus and hence are comparably excited. Macek [18] proposed an adiabatic approximation in which the Schrödinger equation for a two-electron system is expressed in hyperspherical coordinates and the angular equation in the angles $r_{1}, r_{2}$ and $\alpha$ is solved at fixed radius $R$. The eigenvalues of this angular equation represent radial potentials $U_{\mu}(R)$ that describe the radial motion, where $\mu$ is a channel index.

The essential point of Macek's work [18] is that the radial potentials $U_{\mu}(R)$ explain at a glance the key features of the observed He spectra near the $\mathrm{He}^{+}(n=2)$ threshold. Macek found that there are three ${ }^{1} \mathrm{P}^{\mathrm{O}}$ channels $\mu$, designated $\mu={ }^{1} \mathrm{P}^{\mathrm{O}_{+}}, \mu={ }^{1} \mathrm{P}^{\mathrm{O}}$, and $\mu={ }^{1} \mathrm{P}^{\mathrm{O}}(\mathrm{d})$. These three channels have potentials $U_{\mu}(R)$ with very different centrifugal potential barriers when $R \rightarrow 0$. 


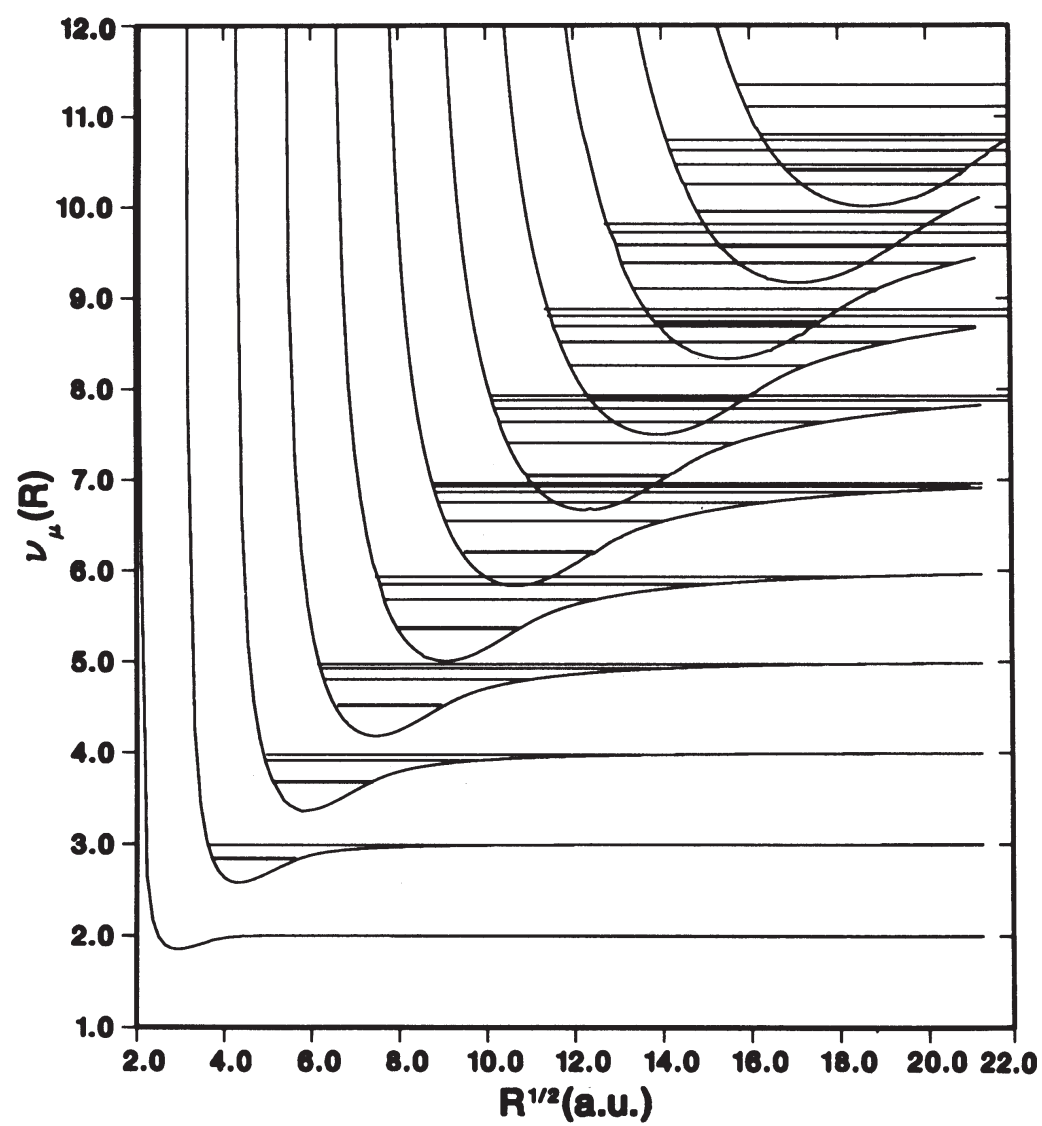

Figure 1. Adiabatic hyperspherical potentials for the lowest ${ }^{1} \mathrm{PO}^{\mathrm{O}_{+}}$channels of $\mathrm{H}^{-}$plotted as effective quantum numbers $v_{\mu}(R) \equiv\left[-2 U_{\mu}(R)\right]^{1 / 2}$ versus $R^{1 / 2}$. Doubly excited state level positions supported within each potential are indicated by horizontal lines. (From Reference 19.)

The intensities of the expected three series are determined by these radial potential barriers because they control the penetration of the excited channel radial wave function in the small $R$ region where the ground-state radial wave function is localized. The smaller the penetration, the less likely is significant overlap with the ground state, and hence the smaller will be the magnitudes of the dipole transition amplitudes which govern the observed intensities. Furthermore, the two potentials having the weakest centrifugal potential barriers, i.e., the " + " and " - " adiabatic hyperspherical potentials, support states that correspond to the $\operatorname{He}(2 s n p \pm 2 p n s)$ ${ }^{1} P^{O}$ states postulated by Cooper et al., [17] thus confirming their interpretation.

The recent experimental work on $\mathrm{H}^{-}$and on He doubly excited spectra near $n>2$ ionization thresholds requires a generalization of this theoretical picture, however. The reason is that in general there are more than one "+" type series converging to higher thresholds. While the selection of those " + " type potentials $U_{\mu}(R)$ having the weakest centrifugal potential barriers turns out to be the key to this generalization, the various centrifugal barriers are so much closer in energy at these higher thresholds that another explanation of the intensities of the observed spectra is called for. Consider first the case of $\mathrm{H}^{-}$.

Sadeghpour and Greene [19] calculated the adiabatic hyperspherical potential curves for very highly excited states of $\mathrm{H}^{-}$converging to $\mathrm{H}(n \leq 12)$. Keeping only the lowest "+" states converging to each threshold $\mathrm{H}(n)$, they were able to interpret the doubly excited resonance structures converging to the $n=4-8$ thresholds that were observed in the photodetachment 
measurements of Harris et al. [14] Sadeghpour and Greene [19] interpreted these observed resonances as the doubly excited states supported by the lowest "+" adiabatic hyperspherical radial potentials, as shown in Figure 1.

The interpretation given by Sadeghpour and Greene for the observed photodetachment spectra [14] implies that all of the other allowed levels supported by the many other ${ }^{1} P^{O}$ adiabatic hyperspherical potentials converging to each $\mathrm{H}(n)$ threshold are not populated in the photodetachment process. Sadeghpour and Greene justified their interpretation by noting that the states corresponding to the lowest + channels converging to each $\mathrm{H}(n)$ threshold have no nodes in the angle $\theta_{12}$ between the two electrons. For example, Figure 2 shows the adiabatic hyperspherical two-electron density [20] as a contour plot in $\theta_{12}$ and $\alpha$ for the two lowest + channels converging to the $\mathrm{H}(n=6)$ threshold. One can see clearly that the density plot for the lowest + channel in Figure $2 \mathrm{a}$ has no $\theta_{12}$ nodes, whereas the plot for the next higher + channel in Figure $2 b$ has a node along $\theta_{12} \approx 0.75 \pi$. Sadeghpour and Greene therefore postulated the propensity rule that in photodetachment of the $\mathrm{H}^{-}$ground state, only doubly excited states having no $\theta_{12}$ nodes are populated with significant intensity.

Sadeghpour and Greene used the bending vibrational quantum number $v$ to quantify the number of nodes in $\theta_{12}$. They postulated that, in general, photoexcitation processes for the ground state of two-electron systems obey the rule, $\Delta v=0$, reasoning that nonadiabatic couplings of transitions from the lowest + channel to higher + channels with $v>0$ are negligible because of the different nodal structures. Note that Rost et al. [21] have pointed out that these nodal structures can be alternatively described in the separable spheroidal coordinates of the molecular orbital picture of two-electron systems. [22,23]

Very recently, Sadeghpour et al. [24] have carried out eigenchannel $R$-matrix calculations of the photodetachment cross sections for $\mathrm{H}^{-}$with excitation of the $n=2,3$, and 4 levels of $\mathrm{H}$. These calculations give quantitative confirmation of the propensity rules postulated in Reference 19 on the basis of the adiabatic hyperspherical model.

The interpretation of the recent He doubly excited state spectrum is similar. Domke et al. [15] reported a high-resolution photoionization study of the doubly excited He states below the $n=2-7$ thresholds of $\mathrm{He}^{+}$. Sadeghpour [25] has shown that the adiabatic hyperspherical representation for highly excited states of He gives a picture similar to that for $\mathrm{H}^{-}$. Namely, the energy levels of doubly excited states calculated in the lowest + adiabatic hyperspherical potentials agree very well with the positions of the experimentally observed [15] resonances for the lowest $n$ levels. Furthermore, the density plots for He display the same kinds of nodal structures, leading to the same conclusion that $\Delta v=0$ is a good propensity rule. For higher $n$ levels, beginning at about $n=6$, overlapping of Rydberg levels corresponding to different $n$ manifolds requires, however, explicit treatment of nonadiabatic coupling terms.

Thus, the newly obtained high-resolution data on doubly excited state spectra of $\mathrm{H}^{-}$and He are permitting theory to develop new understanding of the dynamics of these fundamental three-body Coulomb systems.

\subsection{The Xe 5s-Subshell Photoelectron Angular Distribution}

A particularly striking example of the effect of relativistic interactions is provided by the photoelectron angular distribution for the $5 s$ subshell in xenon. Since xenon is a closed-shell atom, its ground state is spherically symmetric. Hence, the only symmetry axis in electric dipole transitions from the ground state is provided by the polarization vector of the incident light. If one assumes only single configurations to describe initial and final states, then photoionization of the $5 s$ subshell may be represented as the following process:

$$
\text { Xe } 5 s^{2} 5 p^{6}\left({ }^{1} S_{0}\right)+\gamma \rightarrow \operatorname{Xe}^{+} 5 s 5 p^{6}\left({ }^{2} S_{1 / 2}\right) \varepsilon p^{2 S+1} P_{\mathrm{J}=1}
$$

In the absence of relativistic interactions the spin $S$ of the final state in equation (11) is conserved in this transition and hence has the value $S=0$. In this case there is only a single final state channel allowed and hence the asymmetry parameter $\beta$ becomes a constant, independent 

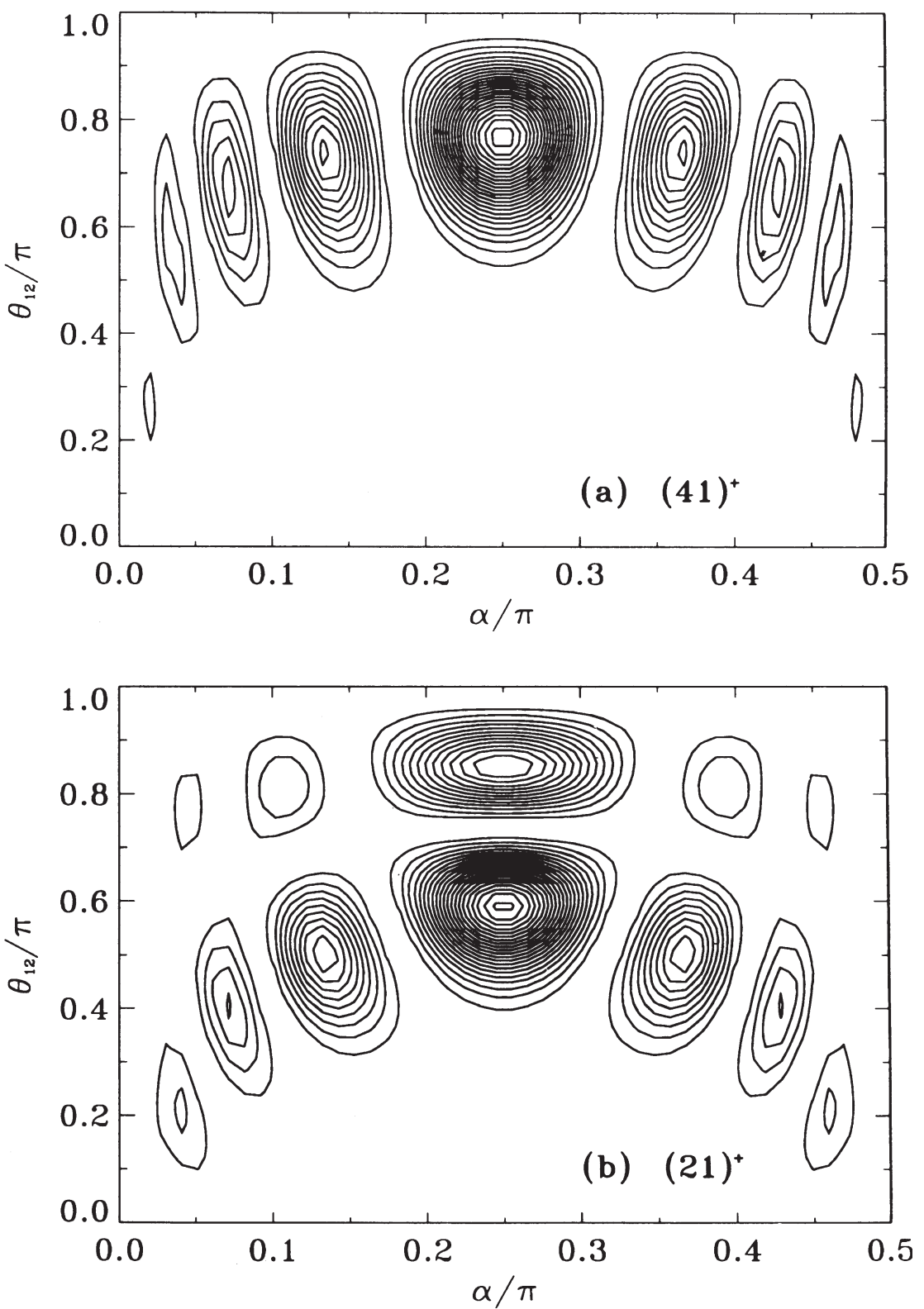

Figure 2. The adiabatic hyperspherical two-electron density function shown as a contour plot versus $\alpha$ and $\theta_{12}$, displaying the nodal patterns for the two lowest + channels in the $n=6$ manifold at $R=80$ a.u. (a) and (b) correspond, respectively, to $(K T)^{A}=(41)^{+}$and $(21)^{+}$channels, i.e., $\mathrm{v}^{A}=0^{+}$and $1^{+}$. (From Reference 19.)

of the photon energy.[11] The value of this constant is $\beta=2$, which implies that the photoelectron has a $\cos ^{2} \theta$ angular distribution about the direction of linear polarization in the case of linearly polarized light.[11]

The first experimental measurement reported by Dehrner and Dill [26] found, however, that $\beta=1.4$ at the photon wavelength $584 \AA$. It was noted that relativistic interactions were likely responsible for this drop in the value of $\beta$ below the nonrelativistically expected value, $\beta=2$. In terms of equation (11), one may note that such relativistic interactions permit the $S=1$ (trip- 
let) channel to have a nonzero amplitude. Within the electric dipole approximation the photoelectron angular distribution asymmetry parameter is then given by

$$
\beta=(2-r) /(1+r)
$$

where $r=\sigma\left({ }^{3} P\right) / \sigma\left({ }^{1} P\right)$. Here $\sigma\left({ }^{3} P\right)$ and $\sigma\left({ }^{1} P\right)$ are the photoionization cross sections for the ${ }^{1} P$ and ${ }^{3} P$ transitions. In the absence of relativistic interactions, $\sigma\left({ }^{3} P\right)$ is zero, and equation (4) shows that $\beta$ is equal to 2 . In practice, $\sigma\left({ }^{3} P\right) \ll<\sigma\left({ }^{1} P\right)$, so that $\beta$ is close to 2 except in the region of the wellknown, near-threshold minimum in $\sigma\left({ }^{1} P\right)$. [Note that $\sigma\left({ }^{1} P\right)$ never becomes exactly zero because of interchannel interactions with photoelectrons from the $5 p$ and $4 d$ subshells, among others.]

The Dehmer and Dill result [26] stimulated much theoretical work to account for these relativistic interactions. The Dirac-Fock (DF) result of Ong and Manson [27], as well as the two relativistic random-phase approximation (RRPA) results of Johnson and Cheng [28], which included coupling between the $5 s$ - and $5 p$-subshell channels and between the $4 d-, 5 s-$, and $5 p$-subshell channels, respectively, all passed close to the single experimentally measured point.[26] These three calculations made very different predictions, however, for the location of the minimum in $\beta$, which is apparently very sensitive to the electron correlations included in the calculation. Only the most detailed calculation, the RRPA $(4 d+5 s+5 p)$ one, was in agreement with the next measured experimental points at lower energy of White et al. [29] The K-matrix results of Huang and Starace [30], which treated the effect of final-state spin-orbit interactions in the Breit-Pauli approximation within a basis of HF nonrelativistic wave functions, did not give nearly so large a drop in $\beta$ as did the other, purely relativistic calculations. $[27,28]$ This result appeared to indicate the importance of having a fully relativistic treatment. The state of affairs as of 1980 is summarized in Figure 3.

The excellent agreement of experiment with the most detailed theoretical predictions (see Figure 3) seemed to provide further evidence that the essential physics of atomic photoionization, at least for rare gas atoms, was well understood. However, this confidence was undermined in 1983 by two new experimental measurements which explored the energy region of the minimum in the Xe 5s-subshell cross section.[31,32] These measurements found a significantly higher value for $\beta$ in the energy region of the minimum, as well as a somewhat lower value for $\beta$ at higher energies, than predicted by the most detailed (RRPA) calculation.[28]

Wendin and Starace [33] postulated a reason for these unexpected discrepancies between theory and experiment, not only for the $\beta$ parameter, but also for the partial cross sections both near threshold and at higher energies. They noted that there is a usually weak interaction, besides the spin-orbit interaction, which has typically been ignored when describing photoionization processes theoretically, but which may have measurable effects when the dominant photoionization transition amplitude is small: final-state ionic configuration interaction. Specifically, the excited $5 s^{2} 5 p^{4}\left({ }^{1} D\right) 5 d\left({ }^{2} S\right)$ configuration in $\mathrm{Xe}^{+}$is very strongly mixed [34-37] with the usual ionic configuration $5 s^{1} 5 p^{6}\left({ }^{2} S\right)$. In a two-level theoretical treatment of process (11), the ionic Hamiltonian would be diagonalized to obtain two new eigenstates, each represented as a linear combination of the configurations $5 s 5 p^{6}\left({ }^{2} S\right)$ and $5 s^{2} 5 p^{4}\left({ }^{1} D\right) 5 d\left({ }^{2} S\right)$. The eigenstate with the lower energy would be a better representation for the ionic state than the single configuration $5 s 5 p^{6}\left({ }^{2} S\right)$. One effect of such ionic configuration mixing in process (11) would be on the kinetic energy and wave function of the continuum electron, which would see both a lower ionization threshold and a less attractive ionic ground state. Another effect would be the modification of the $5 s-5 p$ intershell interaction, which produces the drop in value of the $\beta$ parameter. To the extent that the ratio $\sigma\left({ }^{3} P\right) / \sigma\left({ }^{1} P\right)$ decreases in the neighborhood of the minimum in $\sigma\left({ }^{1} P\right)$ as a result of these two effects, this configuration mixing, they suggested, might explain the discrepancy between theoretical and experimental values for $\beta$.

It was Tulkki [38] in 1989 who showed, in a tour-de-force multichannel, multiconfiguration Dirac-Fock calculation, that the $5 p^{4} 5 d\left({ }^{2} S\right)$ excited ionic configuration was essential to describe the Xe 5 s-subshell photoelectron cross section and angular distribution. He first carried out a configuration interaction calculation for the $\mathrm{Xe}^{+} 5 s^{-1}(J=1 / 2)$ configuration with the five $j j$ - 


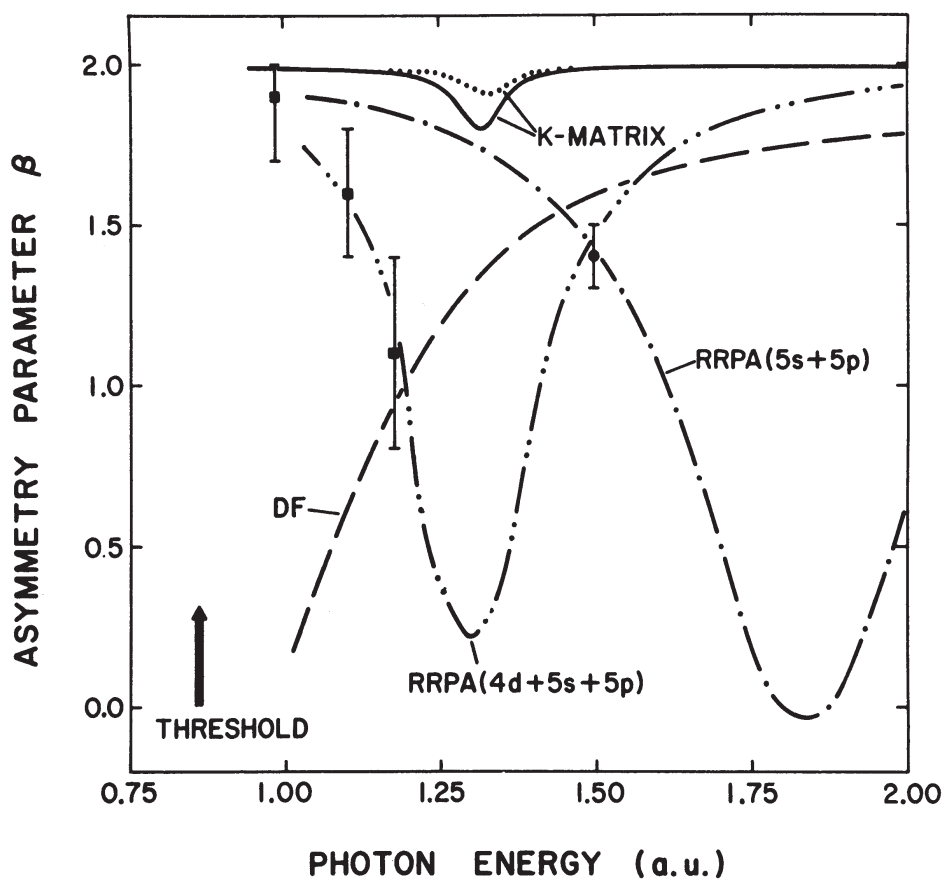

Figure 3. Photoelectron angular distribution asymmetry parameter $\beta$ for the 5 s subshell of xenon. DF: Dirac-Fock results of Ong and Manson. [27] RRPA: relativistic random-phase approximation results of Johnson and Cheng [28], including interchannel correlations between the $5 s$ - and $5 p$-subshell channels (dash-dot line) and between the $4 d-, 5 s-$, and $5 p$-subshell channels (dash-double dot line). K-matrix: results of Huang and Starace [30] including final-state spin-orbit interactions and coupling between the 5s- and $5 p$-subshell channels in dipole length (dotted line) and dipole velocity (solid line) approximation. Solid circle •: Experimental result of Dehmer and Dill [26]. Solid squares घ: Experimental results of White et al. (From Reference 11.)

coupled configurations $\mathrm{Xe}^{+} 5 p^{-2} 5 d(J=1 / 2)$. Then final-state interactions were treated among the 23 channels corresponding to these six ionic states as well as those corresponding to $4 d$ and $5 p$ hole states. For comparison, calculations were also carried out which included only single-excitation channels corresponding to final $4 d, 5 s$, and $5 p$ hole states (13 channels) and only $5 s$ and $5 p$ hole states (7 channels).

Tulkki's results [38] are given in Figure 4, which shows that his 23-channel calculation including final-state ionic configuration interaction is in excellent agreement with the new experimental results for $\beta[31,32]$ as well as with experimental results for the Xe 5 s-subshell cross section.[32, 39-41] This contrasts with the results of his 13- and 7-channel "reference" calculations, which do not include final-state ionic configuration interaction effects. In Tulkki's words,

The effect of interchannel interaction becomes decisive at low photon energies where the cross section and especially the $\beta$ parameter are increased, when the double-excitation channels [i.e., those produced by final state ionic configuration interaction] are included .... [We conclude] that the behavior of the Xe $5 s$ cross section in the threshold region is largely determined by the interaction between the $5 s$ single-hole and $5 p^{4} 5 d$ double-hole ionization channels.

In this way, Tulkki's large-scale calculation demonstrated conclusively that the new physics required to describe process (11) was well understood. Curiously, these new theoretical results [38] and the new experimental results [31,32] lie significantly higher than the original measurement [26], at $584 \AA$, which stimulated all of the succeeding intensive theoretical and experimental efforts. 


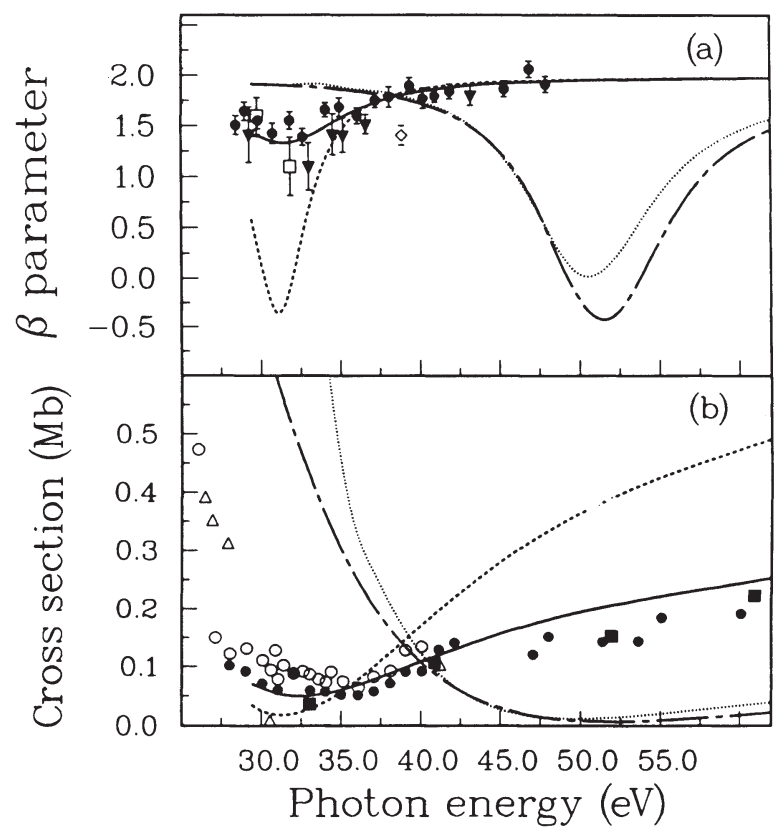

Figure 4. Theoretical and experimental cross section and photoelectron asymmetry parameter for Xe $5 \mathrm{~s}$ photoionization. Theoretical curves of Tulkki [38]-Solid line: 23-channel calculation; dashed line: 13channel calculation; long-short-dashed line: 7-channel calculation; dotted line: 7-channel calculation excluding relaxation. Experimental data-Solid dots: Reference 32; open circles, Reference 39; solid squares, Reference 40; open squares: Reference 29; solid inverted triangles: Reference 31; open triangles: Reference 41; diamonds: Reference 26. (From Reference 38.)

\subsection{Final Ionic State Branching Ratios}

As we have just seen in the case of Xe 5 s-subshell photoionization, configuration interaction effects are very important for describing inner-shell photoionization processes quantitatively. Nevertheless, often a single-configuration point of view allows one to provide a simple interpretation of experimental results. We give here two examples.

3.3.1. Resonant Photoionization of the Be 1s Subshell. In examining the decay of photon-produced Be $1 s 2 s^{2} n p\left({ }^{1} P\right)$ for $n=2,3$, Caldwell et al. [42] found that the predominant decay mode is to the final excited ionic state $\mathrm{Be}^{+} 1 s^{2} n p$, rather than to the ground ionic state, $\mathrm{Be}^{+} 1 s^{2} 2 \mathrm{~s}$. For $n$ $=2$, the $2 p$ ionic state accounts for $95 \%$ of the total cross section, with $\mathrm{Be}^{+} 1 s^{2} 3 p$ the other main contributor. For $n=3,3 p$ is dominant, again with a very small fraction of $2 s$. The experimental data are shown in Figure 5.

One may understand the large observed ratio of $2 p: 2$ s production for the $n=2$ case using an analogue of the compound nucleus model for resonant nuclear scattering, [43], i.e., assuming the decay of the Be $1 s 2 s^{2} 2 p\left({ }^{1} P\right)$ state is independent of its formation. The relative intensities of the $\mathrm{Be}^{+} 1 s^{2} 2 p\left({ }^{2} P\right)$ and $\mathrm{Be}^{+} 1 s^{2} 2 s\left({ }^{2} S\right)$ final ionic states are then proportional to the squares of the following Coulomb matrix elements:

$$
\begin{aligned}
V_{2 p} & \equiv\left\langle 1 s 2 s^{2} 2 p\left({ }^{1} P\right)\left|\sum_{i>j} \frac{1}{r_{i j}}\right| 1 s^{2} 2 p\left({ }^{2} P\right) \varepsilon s\left({ }^{1} P\right)\right\rangle=-\int d r_{1} \int d r_{2} P_{1 s}\left(r_{1}\right) P_{\varepsilon s}\left(r_{2}\right) r_{>}^{-1}\left[P_{2 s}\left(r_{1}\right) P_{2 s}\left(r_{2}\right)\right] \\
V_{2 s} & \equiv\left\langle 1 s 2 s^{2} 2 p\left({ }^{1} P\right)\left|\sum_{i>j} \frac{1}{r_{i j}}\right| 1 s^{2} 2 s\left({ }^{2} S\right) \varepsilon^{\prime} p\left({ }^{1} P\right)\right\rangle \\
& =\int d r_{1} \int d r_{2} P_{1 s}\left(r_{1}\right) P_{\varepsilon^{\prime} p}\left(r_{2}\right) r_{>}^{-1}\left[P_{2 s}\left(r_{1}\right) P_{2 p}\left(r_{2}\right)-\left(2 r_{<} / 3 r_{>}\right) P_{2 s}\left(r_{2}\right) P_{2 p}\left(r_{1}\right)\right]
\end{aligned}
$$



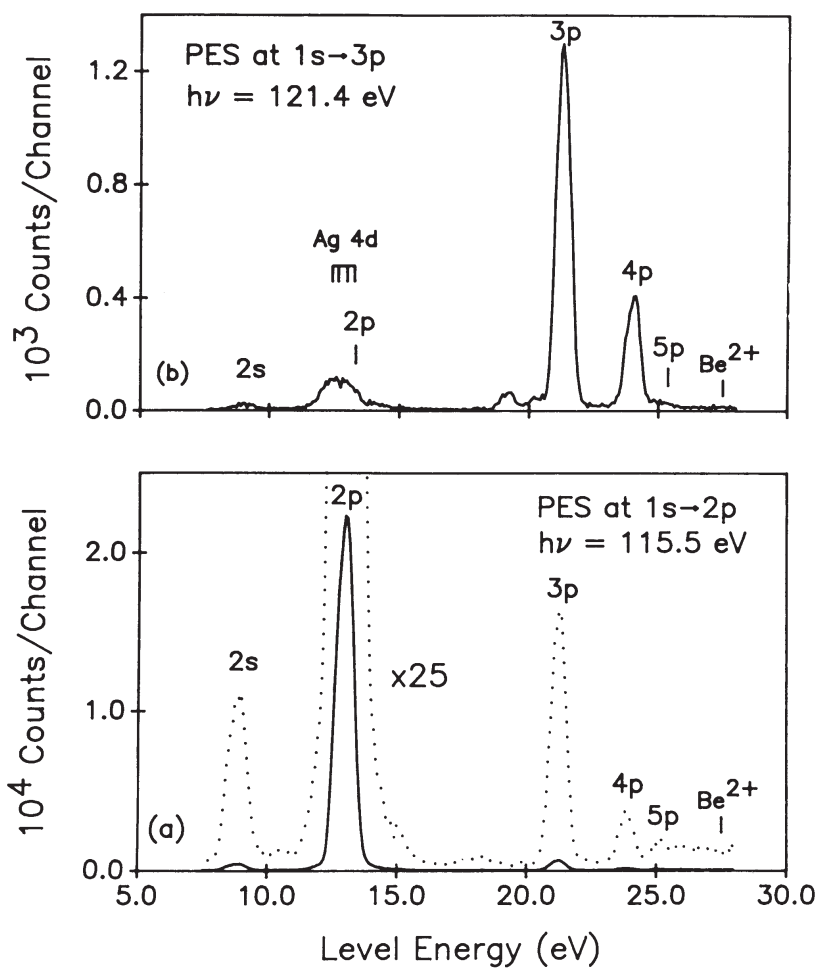

Figure 5. Electron spectra resulting from the decay of the Be $1 s 2 s^{2} n p\left({ }^{1} P\right)$ excited states. (a) $n=2, h \mathrm{v}=115.5$; (b) $\mathrm{n}=3, h \mathrm{v}=121.4 \mathrm{eV}$. The $3 p$ spectrum shows some contamination of the $\mathrm{Be}^{+} 1 s^{2} 2 p$ peak resulting from the presence of silver in the oven. Note the predominance of the $\mathrm{Be}^{+} 1 s^{2} n p, n=2,3$, final states of the ion, and the very small amount of $\mathrm{Be}^{+} 1 s^{2} 2 s$. ( From Reference 42.)

In equations (13) and (14), both initial and final states are described by a single configuration. The right-hand side represents the matrix element in terms of radial Slater integrals over the one-electron radial wave functions $P_{n l}(r) ; \varepsilon$ and $\varepsilon^{\prime}$ are the continuum kinetic energies of the photoionized electron for each of the two final ionic states. Finally, $r_{>} \equiv \max \left(r_{1}, r_{2}\right)$ and $r_{<} \equiv \min \left(r_{1}, r_{2}\right)$.

One sees clearly from (13) and (14) that where the Coulomb repulsion between the jumping electrons is largest, i.e., $r_{1}=r_{2}, V_{2 p}$ has its maximum value [with the factor in brackets $\propto P_{2 s}^{2}(r)$ ] while $V_{2 s}$ has extensive cancellation [with the factor in brackets $\propto P_{2 s}(r) P_{2 p}(r) / 3$ ]. Because of this factor $1 / 3$ in $V_{2 s}$ for $r_{1}=r_{2}$, one expects the squares of (13) and (14) to differ by an order of magnitude.

This expectation is easily confirmed numerically using a basis of bound Hartree-Fock (HF) wave functions calculated for the Be $1 s 2 s^{2} 2 p\left({ }^{1} P\right)$ resonant state as well as HF continuum orbitals $\varepsilon s$ and $\varepsilon p$ calculated in the field of the appropriate relaxed $\mathrm{Be}^{+}$state. Effects of nonorthogonal overlap integrals should also be included. [44] The $1 s, 2 s$, and $2 p$ orbitals are shown in Figure 6. Clearly the region $r_{1}=r_{2}=1.8$ is where $P_{2 s}$ and $P_{2 p}$ have their maximum values. Use of these wave functions leads to a ratio of 24 for the $\mathrm{Be}^{+} 2 p$ to $2 s$ final ionic states, as compared with the experimental branching ratio of $56 \pm 3$.

Matrix elements similar to those in (13) and (14) apply for the resonant state Be $1 s 2 s^{2} 3 p\left({ }^{1} P\right)$. $V_{3 p}$ has the same analysis as $V_{2 p}$. However, the analysis of $V_{2 s}$ requires more care since the first two antinodes of $P_{3 p^{\prime}}$, shown in Figure 6, are both relevant. Such an analysis leads one to expect again that the square of $V_{2 s}$ is at least an order of magnitude smaller than the square of $V_{3 p}$. The HF result reveals it is two orders of magnitude smaller, giving a $3 p: 2 s$ ratio of 250 . This compares with the experimental result of $78 \pm 3$. 


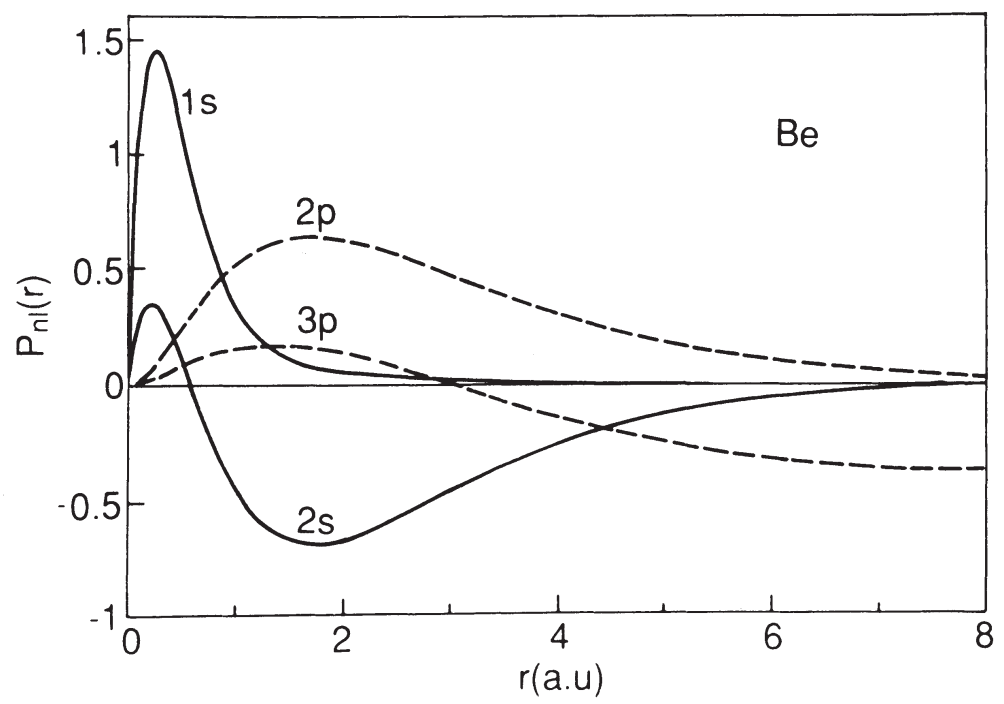

Figure 6. Radial orbital wave functions for $1 s, 2 s$, and $2 p$ in Be calculated in HF approximation for the state Be $1 s 2 s^{2} 2 p\left({ }^{1} P\right)$ The orbital shown is calculated for the state Be $1 s 2 s^{2} 3 p\left({ }^{1} P\right)$. (From Reference 42.$)$

Much more detailed calculations, which include treatment of initial state and final ionic state configuration interactions as well as final state interchannel coupling effects, are needed to obtain quantitative agreement with experiment. [45] This effort is necessary because the $\mathrm{Be}^{+}$ $1 s^{2} 2 s$ ionic state cross section is very small, making its quantitative prediction subject to many competing influences. Clearly, however, the simple single-configuration picture presented above gives a qualitative, order-of- magnitude understanding of why the $\mathrm{Be}^{+} 1 s^{2} 2 s$ ionic state cross section is so small. Namely, the transition matrix element to the $2 s$ state is subject to extensive cancellations, as revealed in (14), whereas the transition matrix elements to the $n p$ states are not, as revealed in (13).

3.3.2. Relative Cross Sections for Fine-Structure Transitions. High-resolution photoelectron spectroscopy experiments [46] can provide the partial cross section for a transition from a particular fine-structure level of an initial atomic state to a particular fine-structure level of the residual ion. Such partial cross sections offer more severe tests of theoretical approximation methods than do the cross sections measured without regard to individual fine-structure levels. However, it is useful to distinguish geometric from dynamic effects particularly since the geometric effects alone can result in intensity distributions that are quite different from those expected for a statistically averaged atomic state.

Theoretical efforts to disentangle geometrical effects from dynamical effects in atomic photoprocesses have a long history. The initial stimuli appear to have been the measurements of Lineberger and co-workers on fine-structure transitions in photodetachment of negative ions in the early 1970s. [47,48] Rau and Fano [49,50] were able to provide interpretations of the observed $[47,48]$ branching ratios for photodetachment of negative ions using a primarily geometrical analysis. The key point of their analysis is that in the region of space where photoprocesses occur, near the origin, the transition amplitudes are unaffected by the generally small spin-orbit and other relativistic interactions that lead to fine structure. Those weak interactions affect primarily the kinetic energy of the electron at asymptotic distances. Hence, the relative magnitudes for particular fine-structure transitions may be calculated analytically using only the geometric (i.e., angular momentum) properties of the transitions. The process of photodetachment is much simpler than that of photoionization since near the threshold for the former process only a single photoelectron orbital angular momentum dominates. In particular, the photodetachment processes considered by Rau and Fano $[49,50]$ involved primarily s-wave 
photoelectrons, which simplifies the theoretical analysis significantly. While the general case of photoionization was sketched in the review of Rau [50], it was not analyzed in detail. However, the work of Fano and Rau on photodetachment stimulated several other researchers to examine the general case of atomic photoionization. [51-55] All of these works make the additional assumption from the start that the dynamical transition amplitudes are independent of the orbital and spin angular momentum quantum numbers relevant to the transition under consideration except for the orbital angular momentum of the photoelectron. In the case that LS-coupled single configurations are used to represent the initial and final states, all of these authors obtain the same results. While the assumption of LS-independent transition amplitudes is not generally a good one, it permits theory to predict the relative cross sections for photoinduced fine-structure transitions completely analytically and hence provides experimenters with benchmarks against which to compare.

Recently, Pan and Starace [56] have presented a more detailed formulation. Within a single-configuration representation of the initial and final states, they have retained the LS-coupling dependence of the dynamical (electric-dipole) transition amplitudes. In special cases (in particular, for the case that the LS-dependence of the radial dipole amplitudes and photoelectron phase shifts is ignored), their results for the partial cross sections for particular fine-structure transitions are shown to reduce to those obtained by others for photodetachment $[49,50]$ and photoionization.[51-55]

The utility of their more general analysis is highlighted by a detailed examination of photoionization of inner $s$ subshells in open-shell atoms, i.e., the process

$$
A n_{0} s^{2} n l^{N}\left(L_{0} S_{0} J_{0}\right)+\gamma \rightarrow A^{+} n_{0} s n l^{N}\left(L_{c} S_{c} J_{c}\right)+e^{-}
$$

When the transition amplitudes are assumed to be LS-independent, then any transition not obeying the triangle inequality,

$$
\left|J_{0}-1 / 2\right| \leq J_{\mathrm{c}} \leq J_{0}+1 / 2
$$

may be shown to have a cross section equal to zero.[56] This selection rule indicates that the photoionization of an $s$ electron removes only an angular momentum of $1 / 2$ from the residual core. However, the more general treatment of Pan and Starace [56], in which the transition amplitudes are assumed to retain their LS-dependence, indicates that no such restriction on the allowed transitions applies: transitions violating the triangle relation in (16) (but satisfying the usual angular momentum and parity conservation laws) are allowed. There is both experimental and theoretical evidence, however, that fine-structure transitions which do not satisfy (16) are nevertheless quasiforbidden, indicating therefore that there are hitherto unsuspected cancellation effects between different transition amplitudes contributing to these particular fine-structure transitions.

Consider the case of atomic chlorine,

$$
\mathrm{Cl} 3 s^{2} 3 p^{5}\left({ }^{2} P_{J_{0}}\right)+\gamma \rightarrow \mathrm{Cl} 3 s 3 p^{5}\left({ }^{3} P_{J_{\mathrm{c}}}\right)+\mathrm{e}^{-}
$$

for which Pan and Starace [56] presented LS-dependent HF results for the relative partial cross sections and compared these to the purely geometric predictions that one may make by assuming that the transition amplitudes are LS-independent. This case was chosen because there exist experimental data [57] for this process and also because Robicheaux and Greene have recently carried out multiconfiguration, eigenchannel $R$-matrix calculations for photoionization of both the $3 p$ subshell [58] and 3s subshell [59] of atomic chlorine. Reference 59 does not contain plots of the fine-structure partial cross sections; however, Robicheaux's results are presented in Reference 56.

The relative partial cross sections for process (17) for the case $J_{0}=3 / 2$ are presented in Figure 7. The purely geometrical predictions, which are obtained by assuming LS-independent dynamical amplitudes, are indicated by the horizontal solid lines. These predictions agree surprisingly well with those obtained using the more general equations of Pan and Starace [56], in which the LS-dependent amplitudes and phase shifts are calculated in HF approximation. These single-configuration predictions are shown in Figure 7 to agree very well also with the 


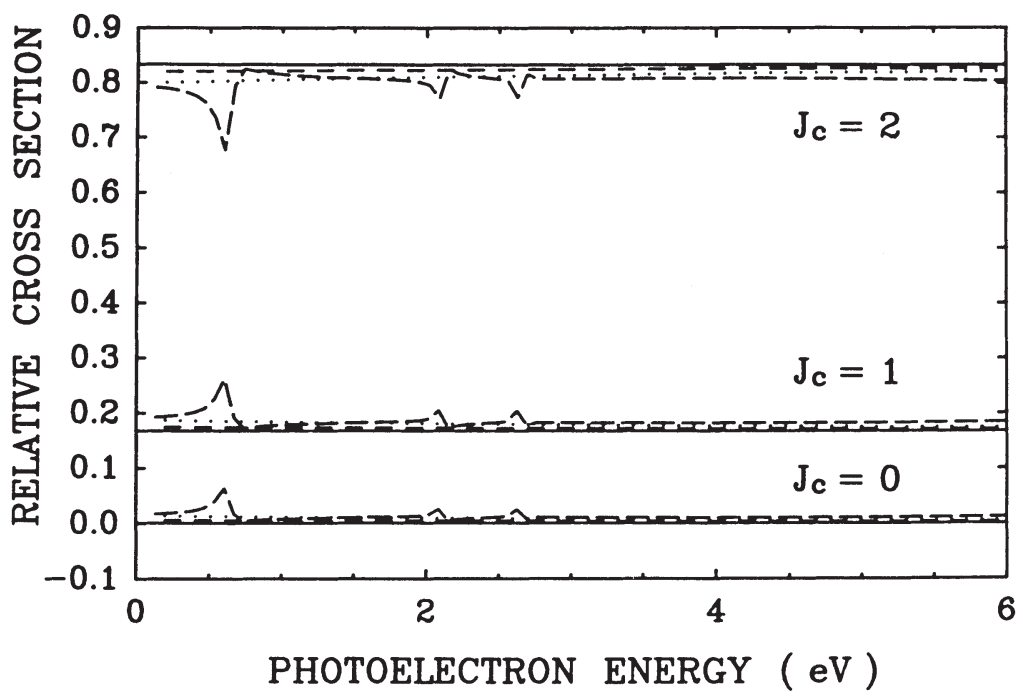

Figure 7. Relative cross sections for the transitions $\mathrm{Cl} 3 s^{2} 3 p^{5}\left({ }^{2} P_{3 / 2}\right)+\gamma \rightarrow \mathrm{Cl}^{+} 3 s 3 p^{5}\left({ }^{3} P_{J_{\mathrm{c}}}\right)+\mathrm{e}^{-}$in three levels of approximation. Solid lines: purely geometrical predictions assuming $L S$-independent transition amplitudes [56]. Short-dashed (dotted) lines: LS-dependent HF predictions in dipole length (velocity) approximation of Pan and Starace [56]. Long-dashed lines: multiconfiguration, eigenchannel $R$-matrix results of Robicheaux and Greene [59]. (From Reference 56.)

multiconfiguration, eigenchannel $R$-matrix results of Robicheaux and Greene [59], except of course in the vicinity of autoionizing resonances. This agreement is all the more remarkable since they find that the $3 P^{O}$ state of the ion only contains $71 \% 3 s 3 p^{5}$ and $29 \%$ other configurations.[59] Furthermore, a recent experiment [57] was unable to discern any signal for the quasiforbidden transition $J_{0}=3 / 2 \rightarrow J_{c}=0$ shown in Figure 7 .

In conclusion, based on comparisons with more general theoretical results [56,59], as well as with experiment [57], it appears that inner s-subshell transitions of the form of (15) which do not obey the triangle inequality in (16) are quasiforbidden. Thus, simple geometrical arguments based on a single-configuration, LS-independent analysis appear to give a reliable indicator of otherwise unsuspected cancellation among the amplitudes contributing to these processes. This is true not only in the case of the more general single-configuration treatment of Pan and Starace [56], but also for a multiconfiguration treatment.[59]

\subsection{Fluorescence Spectroscopy}

High-brightness light sources enable one to study relatively weak processes, such as satellite structures attendant on the main photoionization line. One method of studying the often complex satellite spectrum is fluorescence spectroscopy.[60] It provides complementary information to photoelectron spectroscopy. Its advantages are its ability to study satellite structures near threshold, and its ability to distinguish satellite states having similar energies (since they fluoresce with different wavelengths). Also, it obviates the need for coincidence techniques. A disadvantage is the necessity for cascade corrections.

Fluorescence spectroscopy has had in recent years a number of successes in elucidating photoionization spectra. One of the earliest of such successes was the first clear evidence of doubly excited states converging to the $\mathrm{He}^{+}(n=3)$ threshold by Woodruff and Samson.[61] This experiment measured the $\mathrm{He}^{+} 2 p$ fluorescence decay at $304 \AA$ and saw evidence in this spectrum (as a function of incident photon energy) of helium doubly excited states autoionizing to the $\mathrm{He}^{+}(n$ $=2$ ) state. Also, the $\mathrm{He}^{+} 2$ s population was observed by quenching with an electric field. 
Another such success was the finding by Schartner et al. [60,62] that doubly excited resonance structures are prominent in the region of the threshold for the Ar 3s-subshell photoionization cross section. These findings underlined the need for theoretical calculations to treat such satellite structures in inner-shell photoionization cross section calculations. They also indicated the need for high-brightness synchrotron light sources to enable experiment to measure the line shapes for these resonances.

Very recent work on Ne photoionization by Samson et al. [63] provides a further example of the complementary information gained by fluorescence spectroscopy. Consider the neon photoexcitation process,

$$
\text { Ne } 2 p^{6}\left({ }^{1} S_{0}\right)+\gamma \rightarrow \mathrm{Ne}^{* *} 2 p^{4}\left({ }^{3} P\right) 3 p n l\left({ }^{2 S+1} P\right)
$$

that is followed by the autoionization process

$$
\mathrm{Ne}^{* *} 2 p^{4}\left({ }^{3} P\right) 3 p n l\left({ }^{2 S+1} P\right) \rightarrow\left(\mathrm{Ne}^{+}\right) * 2 p^{4}\left({ }^{3} P\right) 3 p\left({ }^{4} P\right)+\mathrm{e}^{-}
$$

By angular momentum selection rules, process (19) is only possible for the triplet doubly excited state (i.e., $S=1$ ). This state is produced in process (18) only through final-state spin-orbit interactions; in LS-coupling, the triplet state is forbidden by electric dipole selection rules.

In a photoelectron spectroscopy experiment [64], it is difficult to distinguish the $\left(\mathrm{Ne}^{+}\right)^{*} 2 p^{4}\left({ }^{3} P\right) 3 p\left({ }^{4} P\right)$ satellite from the $\left(\mathrm{Ne}^{+}\right)^{*} 2 p^{4}\left({ }^{1} D\right) 3 s\left({ }^{2} D\right)$ satellite since the two states differ in energy by only a few millielectron volts. Fluorescence spectroscopy, however, permits one to distinguish the ${ }^{4} P$ satellite by its fluorescence in the wavelength range $3665-3778 \AA$, i.e.,

$$
\left(\mathrm{Ne}^{+}\right)^{*} 2 p^{4}\left({ }^{3} P\right) 3 p\left({ }^{4} P_{J}\right) \rightarrow\left(\mathrm{Ne}^{+}\right)^{*} 2 p^{4}\left({ }^{3} P\right) 3 s\left({ }^{4} P_{J^{\prime}}\right)+\gamma^{\prime}
$$

where the wavelength range arises from transitions between the various allowed $J$ and $J^{\prime}$ levels. In contrast, the $\left(\mathrm{Ne}^{+}\right)^{*} 2 p^{4}\left({ }^{1} D\right) 3 s\left({ }^{2} D\right)$ satellite fluoresces in the neighborhood of $406 \AA$.

A comparison of the photoelectron [64] and fluorescence [63] spectra is shown in Figure 8. The bottom panel shows the fluorescence spectrum [63] - see process (20) - as a function of the incident photon energy-see process (18). The top panel shows the corresponding photoelectron spectroscopy (PES) spectrum [64], which cannot distinguish between the $3 p\left({ }^{4} P\right)$ and $3 s\left({ }^{2} D\right)$ satellites. It is plotted also versus incident photon energy. Notice that the fluorescence spectrum is obtained at the very threshold for producing the $3 p\left({ }^{4} P\right)$ satellite, whereas the PES spectrum starts about $0.35 \mathrm{eV}$ above threshold.

Clearly, fluorescence spectroscopy is a valuable complementary tool for studying detailed features of photoionization spectra.

\subsection{Photoionization of Inner Shells of Excited States}

Inner-shell photoionization differs from outer-shell processes in that the relaxation of the ion with an inner-shell vacancy is considerably more complex, even aside from the Auger or xray processes which fill the vacancy. From a physical point of view, removal of an outer-shell electron strongly affects the other outer-shell electrons which it partially screens. But, if the outer-shell electron is thought of as roughly a shell of charge at the outer-shell radius, Gauss's law of electrostatics tells us that this electron exerts no force inside the shell, i.e., on inner-shell electrons. Thus, the primary effect of the removal of an outer-shell electron on inner shells is a constant change in the potential energy and little else.

Removing an inner-shell electron, on the other hand, changes the screening (and, thus, the potential) of all electrons in that shell and in all shells outside it, thereby leading to much more significant relaxation effects. The effect is likely to be even more pronounced for an excited electron since more of the electron density is outside the inner shell and removal of an innershell electron changes the screening by close to a full electron charge; the more highly excited the electron, the greater the change in screening.

As an example, $2 p$ inner-shell photoionization has been investigated experimentally [65] for both ground-state sodium atoms $\left(1 s^{2} 2 s^{2} 2 p^{6} 3 s\right)$ and excited-state sodium atoms $\left(1 s^{2} 2 s^{2} 2 p^{6} 3 p\right)$. As 


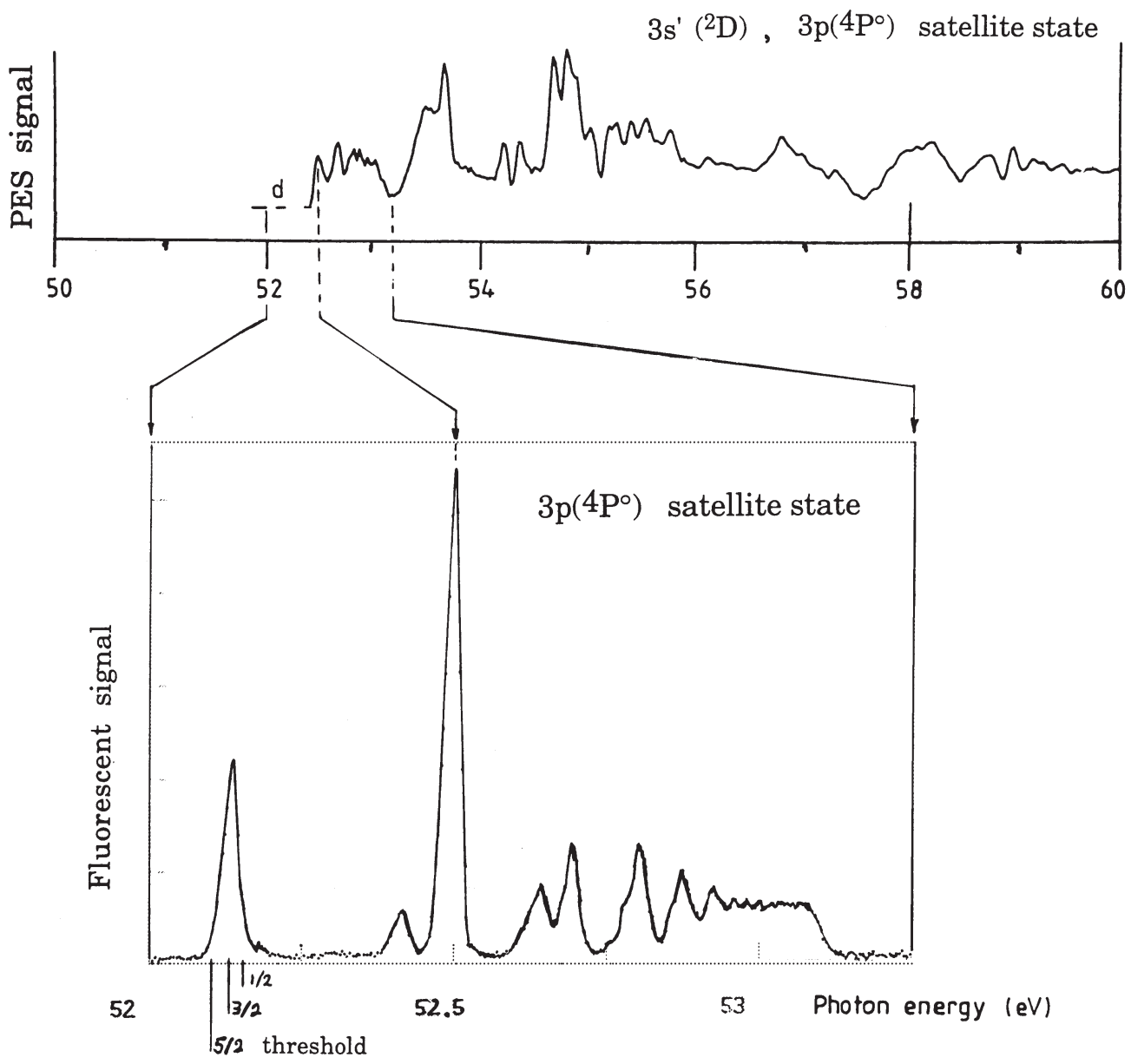

Figure 8. Comparison of photoelectron spectrum resulting from autoionization of the doubly excited states $\mathrm{Ne}^{* *} 2 p^{4}\left({ }^{3} P\right) 3 p n s, d$ (top figure, from Reference 64) with the fluorescence spectrum of the $\left(\mathrm{Ne}^{+}\right)^{*} 2 p^{4}\left({ }^{3} P\right) 3 p\left({ }^{4} P^{O}\right)$ satellite state (bottom figure, from Reference 63). Both figures are plotted versus initial photon energy - see process (18). For further details, see text description.

a practical matter, relaxation shows up as satellite transitions, i.e., photoionization processes where one electron is ionized and another is excited. The intensity of the satellites for $2 p$ ionization from the ground state, relative to the main transition in which all electrons other than the photoelectron remain spectators, is shown in Figure 9. As a function of energy, the ratio remains relatively constant, just below 20\%. Also shown in Figure 9 is the corresponding ratio for the $3 p$ excited state of $\mathrm{Na}$; it is also independent of energy, but about $40 \%$. Thus, the excitation of the $3 s$ electron to $3 p$ in the initial state of Na roughly doubles the cross section for satellite transitions.

These results can be understood in terms of changes in screening, as discussed above. The cross section for the main transition for $2 p$ photoionization in the presence of an outer $n l$ spectator electron is proportional to $\left|\left\langle n l_{\mathrm{i}} \mid n l_{\mathrm{f}}\right\rangle\right|^{2}$, where the subscripts " $\mathrm{i}$ " and " $\mathrm{f}$ " refer to the initial and final states, respectively. This overlap factor, which differs from unity because of relaxation, is about 0.85 for $3 s$ and 0.7 for $3 p$, leading to the results shown in Figure 9. Since $3 s$ is such a penetrating orbital, removal of a $2 p$ electron does not have as large an effect as for the $3 p$ which has a larger radius and which is much less penetrating. Thus, relaxation is more significant for the $3 p$ initial state, leading to the enhanced satellite intensity, in agreement with theoretical predictions.[66] 


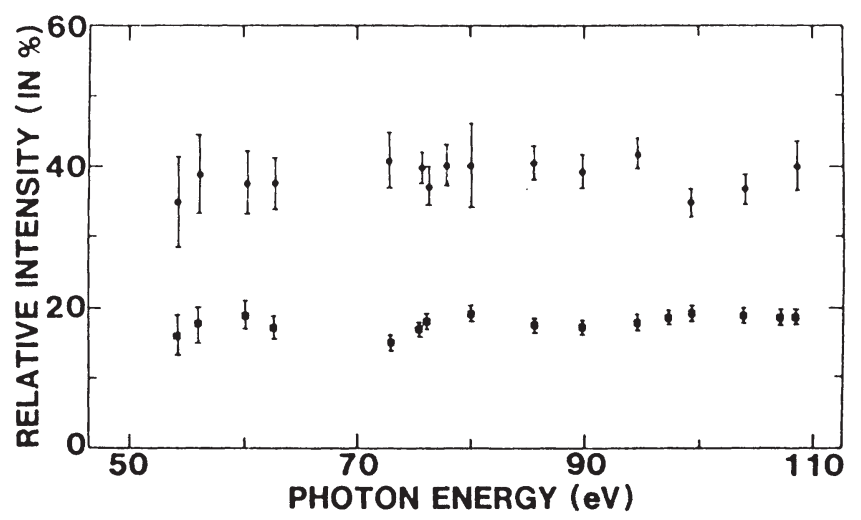

Figure 9. Variation, as a function of photon energy, of the relative intensity of shakeup satellites for sodium atoms in the ground state $\left(\boldsymbol{\square}=2 p^{5} 4 s\right.$ final ionic states) and in the $3 p$ excited state $\left(\bullet=2 p^{5} 4 p\right.$ final ionic states), respectively. (From Reference 65.)

Clearly, by these arguments, this relaxation effect will be even more pronounced for more highly excited states. A recent calculation [67] investigated inner-shell photoionization for excited states of lithium. The results for $1 s^{2} 3 p$ are given in Figure 10, which shows the cross sections for leaving the $\mathrm{Li}^{+}$ion in the $1 s 2 p, 1 s 3 p$, and $1 s 4 p^{3,1} \mathrm{P}$ states. The outstanding feature of these results is that the dominant cross section is that of the $1 s^{2} 3 p\left({ }^{2} P\right) \rightarrow 1 s 4 p\left({ }^{3} P\right)+\mathrm{e}^{-}$channel, which results from an ionization plus satellite excitation transition. The next largest cross section is for the $1 s^{2} 3 p\left({ }^{2} \mathrm{i}\right) \rightarrow 1 s 4 p\left({ }^{1} P\right)+\mathrm{e}^{-}$transition, which also involves ionization plus excitation. In fact, away from threshold, the $4 p$ "satellites" represent $76 \%$ of the total cross section, whereas the $3 p$ "main" transitions account for only $13 \%$. As in the case of sodium, the explanation is the relaxation of the $3 p$ orbital of the initial state, as reflected in the overlap factors.

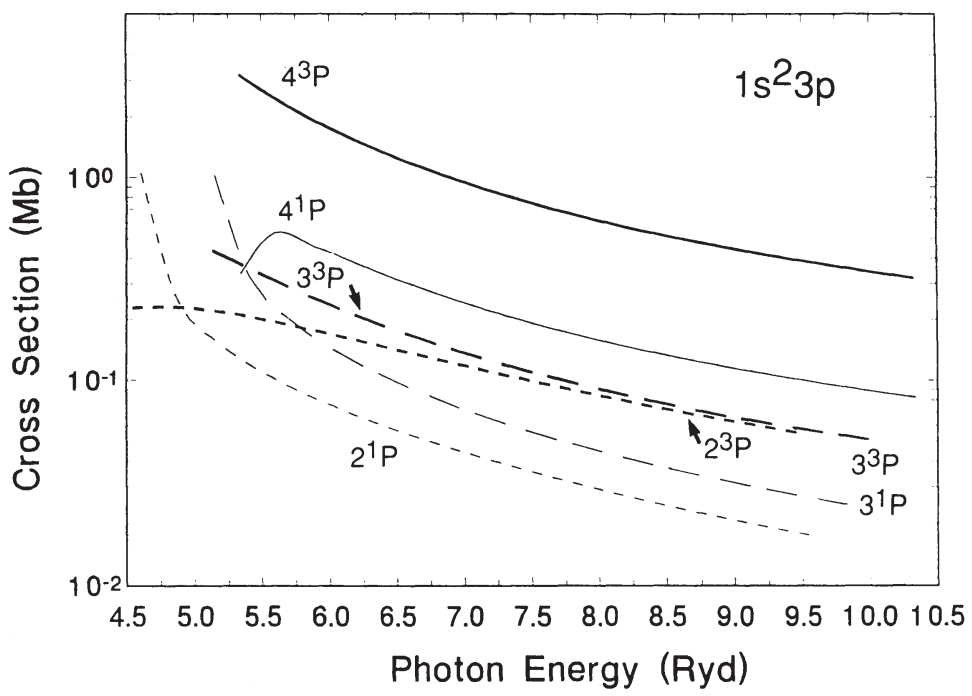

Figure 10. Photoionization cross sections calculated for $1 s$ ionization from the Li $1 s^{2} 3 p$ excited state to various $n^{3} P$ and $n^{1} P$ states of the $\mathrm{Li}^{+}$ion plotted versus photon energy. The curves are the dipole-length results, which are in good agreement with the dipole velocity results (not shown). (From Reference 67.) 
That the overlaps favor $3 p \rightarrow 4 p$ so strongly is related to the fact that a reasonably highly excited state of lithium is virtually completely screened by the $1 s^{2}$ core; the excited electron "sees" a charge of 1 . Similarly, in the $\mathrm{Li}^{+}$ion, the excited electron "sees" a charge of 2 , so that the $3 p_{\mathrm{f}}$ orbital of the final state is considerably more compact than the $3 p_{\mathrm{i}}$ orbital of the initial state. Thus, the principal overlap of the $3 p_{\mathrm{i}}$ wave function is with the $4 p_{\mathrm{f}}$ orbital which occupies the same region of space owing to the increased charge it "sees." One may easily verify this from even the simple Bohr atomic model. For lower excited states, or ground states, the effect is not nearly so dramatic, as seen for sodium, because the change in effective charge is far smaller than the factor of 2 change that occurs for highly excited states.

While this phenomenon has not yet been seen experimentally for highly excited states, the work on sodium $[65,68,69]$ and more recent work on potassium [70] are certainly indicative of it. In addition, it has been observed experimentally in multiphoton ionization of excited barium [71] as well as in autoionization of core-excited argon [72]. In any case, with the emergence of the technology to create and ionize such excited states [73,74], one has a new "laboratory" in which to study multielectron transitions.

\subsection{Photoelectron Angular Distributions from Parity-Unfavored Transitions}

Photoelectron angular distributions give in general information about amplitudes and phases of the dipole matrix elements involved in the photoionizing transitions. The parameter $\beta$ [equation (7)], which describes the angular distribution, is normally energy-dependent since the amplitudes and phases of the matrix elements are always energy-dependent. However, the energy-dependence of $\beta$ arises from the interference of the transition amplitudes for alternative photoelectron emission channels, analogous to the interference underlying a double slit experiment. In cases where there is only a single photoelectron emission channel, no interference is possible and $\beta$ must be independent of energy. Such a situation occurs in the photoionization of the ground state of the hydrogen atom, or any alkali atom, if relativistic effects are ignored. In these cases, there is only an $s \rightarrow p$ transition and $\beta=2$. This corresponds to a $\cos ^{2} \theta$ distribution, which attains its maximum in the direction of the photon's electric vector. This result is consistent with the intuitive picture of photoionization as a response to the impulse of the photon's electric field. In addition, in certain cases, $\beta$ may have the constant value 0.2. [11]

Most curious, however, is the prediction that under certain conditions, $\beta$ can take the energy-independent value of -1 , giving rise to a $\sin ^{2} \theta$ distribution. This distribution attains its maximum at $90^{\circ}$ to the photon's electric vector, an entirely counterintuitive result. In the language of the angular momentum transfer analysis of photoelectron angular distributions, this is called a "parity-unfavored" transition, discussed in Section 2, where it was seen that such a transition is characterized by

$$
\pi_{0} \pi_{\mathrm{c}}=-(-1)^{j_{\mathrm{t}}}
$$

where $j_{\mathrm{t}}$ is the angular momentum transfer and $\pi_{0}$ and $\pi_{\mathrm{c}}$ are the parities of the initial atomic and the final ionic states, respectively. In many photoionization processes, the possible values of $j_{t}$ include one or more which are parity-unfavored. [11] But transitions which are purely parity-unfavored were thought to be relatively rare. For example, the transition

$$
p^{3}\left({ }^{2} D\right) \rightarrow p^{2}\left({ }^{1} S\right) \varepsilon d\left({ }^{2} D\right)
$$

in atomic nitrogen or phosphorus is such a process.[75] Within the context of LS coupling, equation (6) for $j_{\mathrm{t}}$ becomes

$$
\vec{j}_{\mathrm{t}}=\vec{L}_{\mathrm{c}}-\vec{L}_{0}=\vec{j}_{\gamma}-\vec{l}
$$

with $\vec{L}_{0}, \vec{L}_{\mathrm{c}}$, and $\vec{l}$ the orbital angular momenta of the initial atomic state, the final ionic core, and the photoelectron, respectively, while $j_{\gamma}$ is the photon angular momentum (having a magnitude of unity). Thus, for process (22), in which $L_{\mathrm{c}}=0$ and $L_{0}=2$, equation (23) shows that $j_{\mathrm{t}}=2$ 


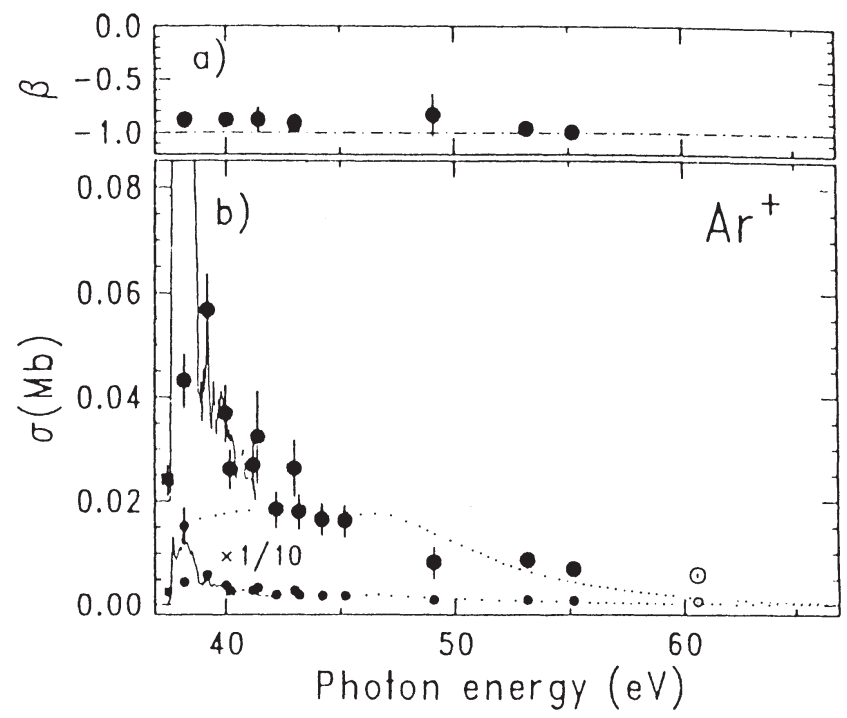

Figure 11. (a) Angular distribution parameter $\beta$ and (b) partial cross section $\sigma$ of the $3 p^{4}\left({ }^{1} D\right) 3 d\left({ }^{2} P\right)$ parityunfavored satellite transition in argon $\left(E_{\mathrm{B}}=37.4 \mathrm{eV}\right)$. The dotted curve represents the $3 p$ partial cross section scaled to the satellite intensity at equal kinetic energy. (From Reference 77.)

is the only possibility. Furthermore, $\pi_{0}=-1$ and $\pi_{c}=1$, so that this is a parity-unfavored transition. Thus, theory predicts that $\beta=-1$. However, experiment has not yet confirmed this result.

Previously, purely parity-unfavored transitions have been investigated experimentally only for autoionizing resonances.[76] Recently, however, it has been pointed out that a whole class of states exist which exhibit this phenomenon $[77,78]$ namely, a subset of satellite transitions where one electron is ionized and another is excited. As an example, consider photoionization of the ground state of argon in which one $3 p$ electron is ionized and another is promoted to a $3 d$ state,

$$
3 p^{6}\left({ }^{1} S\right) \rightarrow 3 p^{4}\left({ }^{1} D\right) 3 d\left({ }^{2} P\right) \varepsilon p\left({ }^{1} P\right)
$$

In this case $L_{0}=0$ and $L_{c}=1$ so that, by equation (23), the only possible value for $j_{\mathrm{t}}$ is $j_{\mathrm{t}}=1$. Then, since $\pi_{0}=\pi_{c}=1$, according to (21) this is a purely parity-unfavored transition. The experimental cross section and $\beta$ parameter are shown in Figure 11, in which it is seen that, although the cross section is highly dependent on the photon energy, $\beta$ is constant at -1 . From this discussion, it is clear that there exist a whole class of such satellite transitions for all of the noble gases, as well as for the rest of the periodic system.

\subsection{Double Photoionization of Helium}

The double photoionization process is particularly interesting since the final state, an ion plus two outgoing electrons, is an example of the three-body continuum Coulomb problem. This is not merely one of the oldest unsolved problems in atomic physics, it is one of the oldest unsolved problems in physics! From a theoretical point of view, the difficulty is that, although the boundary conditions are now known [79], realistic solutions to the Schrödinger equation which conform to these boundary conditions have not been obtained. In addition, in single photoionization, the energy of the photoelectron generated by a photon of a given energy is fixed. In double photoionization, on the other hand, since two electrons are ejected, only the sum of the two energies is fixed. Thus, the total double photoionization cross section is a sum over all of the possible energy sharings between the two electrons. This, of course, makes experimental photoelectron spectroscopy of the double photoionization process considerably more difficult than for the single photoionization process. 
Despite the inherent extra complexity of the double photoionization process, it can be shown that at high photon energies the behavior of the double photoionization cross section for He is proportional to $E^{-7 / 2}$, where $E$ is the total energy of the two photoelectrons.[80] The same asymptotic behavior is predicted for single photoionization. These asymptotic behaviors depend only on the character of the initial state and the fact that the photoionization process is an electric dipole transition.[80] Thus, since both cross sections approach the same asymptotic form at high energy, the ratio of double to single photoionization must also approach a constant limit. But knowing that the ratio approaches a constant limit does not tell us at what energy the asymptotic ratio is reached, or the value of the asymptotic ratio.

Experimental measurements of the ratio of double to single ionization were limited to fairly low energies until recently when it was measured in the range of $2-12 \mathrm{keV}$.[81,82] The ratio was found to be $1.5 \pm 0.2 \%$, and essentially constant over this entire range. This indicates that a constant ratio has been reached. Also, this ratio is in good agreement with recent calculations as well as with a number of earlier ones.[80] The results disagree, however, with a recent prediction, based on an analogy with electron impact ionization, that the ratio should be decreasing in this region, despite remarkable success of that model at low energy.[83a] The difference may indicate the greater importance of shake-off effects as compared to electron scattering effects at high photon energies.[83b]

These high-energy experiments are only possible with high-brightness sources since the cross sections become so small, particularly for double ionization. Furthermore, measurement of the angular and energy correlations between the two photoelectrons produced in double photoionization requires a coincidence measurement, which in turn necessitates a still higher photon intensity. But such detailed measurements are precisely what is needed to shed further light on this fundamental three-body Coulomb problem.

In fact, both experimental and theoretical investigations are continuing at a rapid pace. Among the recent works is a measurement of the double to single ionization ratio with a fairly fine energy grid over a broad energy range [84], which shows good agreement in the intermediate energy region with the recent theoretical work of Pan and Kelly [85]; this indicates that some higher order perturbations, which are effectively included in Pan and Kelly's work [85], but not included in recent calculations of Hino et al. [86], are important. It has also been pointed out that, with increasing energy, the atomic Compton effect becomes an increasingly important contributor to both the single and the double ionization processes, and that it dominates above the $10 \mathrm{keV}$ region.[87] A recent calculation which includes the Compton contribution [88] is in excellent agreement with experiment at the higher energies, but there is some debate as to the high energy Compton ratio. Further, the angular distribution of photoelectrons resulting from double photoionization has been measured $[89,90]$, and good agreement is found with recent calculations.[91] Finally, at the low energies, a very recent experiment [92] using cold ion recoil momentum spectroscopy (COLTRIMS) has found a ratio substantially lower ( 25\%) than previous experiments. This new result also makes the photon ratio consistent with the charged particle impact $\mathrm{He}^{++} / \mathrm{He}^{+}$ratio.[92]

\subsection{Multichannel Aspects of Photoionization}

Photoionization is often thought of as a single electron process, owing to the fact that the transition operator responsible for low-energy photoionizing transitions-the electric dipole operator-is a single particle operator. However, although it is quite true that in many cases the single electron aspects of photoionization are most important, it is equally true that in many cases multielectron aspects dominate.[7] These multielectron aspects arise from the electron-electron interaction and are an example of the general problem of electron correlations.

From a theoretical point of view, electron correlation can be included explicitly, by including the $r_{i j}$ dependence in the wave function, or implicitly, via expansion in a complete basis set. When the initial or final ionic discrete states are expanded, multiconfiguration or configuration interaction $(\mathrm{CI})$ wave functions are formed. Multiconfiguration effects can cause a seri- 


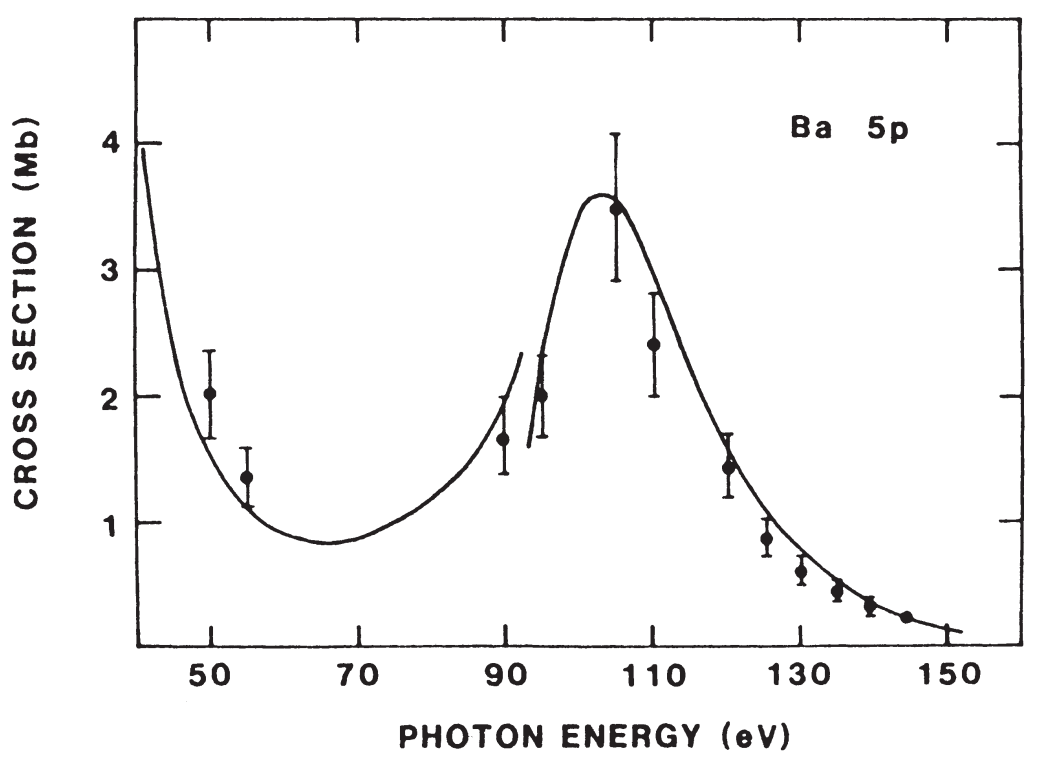

Figure 12. Experimental and theoretical data for the Ba $5 p$ photoionization cross section. (From Reference 93.)

ous breakdown in the single electron picture, as double photoionization (see Section 3.7) amply demonstrates.[80] The total final continuum wave function can also be expanded, and this is referred to as a multichannel expansion. The Coulomb matrix elements between these channel wave functions are known generally as interchannel coupling matrix elements.

The effects of interchannel interactions can be exhibited using simple perturbation theory. Let $\left|f_{0}\right\rangle$ and $\left|f_{1}\right\rangle$ denote the final-state wave functions of two different channels of a photoionization process resulting from the initial state $|i\rangle$. If the Hamiltonian of the system is $H$, and the photoionization transition operator is $T$, then the matrix element for the $|i\rangle \rightarrow\left|f_{0}\right\rangle$ photoionization process, including the effects of interchannel interaction, is given essentially by

$$
\left\langle i|T| f_{0}\right\rangle+\sum_{f_{1}} \frac{\left\langle i|T| f_{1}\right\rangle\left\langle f_{1}|H| f_{0}\right\rangle}{\Delta E}
$$

The first term in (25) is the direct electric dipole transition to the final state $\left|f_{0}\right\rangle$. The second term indicates schematically the indirect transition amplitude resulting from an electric dipole transition to the intermediate states $\left|f_{1}\right\rangle$ followed by an interchannel interaction to the final state $\left|f_{0}\right\rangle$. $\Delta E$ denotes the energy difference between the final state and the intermediate state and $\Sigma_{f}$ denotes a sum over degenerate quantum numbers in the intermediate state $\left|f_{1}\right\rangle$, as well as an integration over intermediate state energy. The question then looms as to when the second term in the above equation has a significant influence relative to the first term. The mixing coefficient $\left\langle f_{0}|H| f_{1}\right\rangle / \Delta E$ will be most significant in general when $\Delta E$ is small; thus, the second term is most important when channels $f_{0}$ and $f_{1}$ are degenerate, i.e., they both are accessible at the given photon energy. Then if the basic single-particle dipole matrix element for channel $1,\left\langle i|T| f_{1}\right\rangle$, is significantly larger than $\left\langle i|T| f_{0}\right\rangle$, the second term in the above equation can be quite important.

As an example, consider the case of Ba $5 p$ photoionization [93], shown in Figure 12. The cross section is dropping from threshold (which is actually below the lowest energy shown in this curve), but then there is a huge hump extending over some $60 \mathrm{eV}$ and centered about $h \mathrm{v}$ $\cong 100 \mathrm{eV}$. This hump is seen to be the major feature of the cross section over a broad energy region, and is the result of interchannel coupling of the $5 p$ channels with the $4 d$ channels.[93] If it were not for this coupling, the $5 p$ photoionization cross section would be a few tenths of 
a megabarn, as can be inferred from Figure 12. The $4 d$-subshell threshold is around $100 \mathrm{eV}$, and its cross section has a maximum near threshold of about $20 \mathrm{Mb}$ [94], which indicates that $\left\langle i|T| f_{1}\right\rangle$ is about an order of magnitude larger than $\left\langle i|T| f_{0}\right\rangle$ at this energy, since the $4 d$ cross section is about two orders of magnitude larger. Thus, the Ba $5 p$-subshell cross section, in this energy range, is dominated by its interchannel coupling with the $4 d$ channels. From a theoretical viewpoint, this occurs because the true wave function of the final continuum state is a mixture of the $5 p \rightarrow \varepsilon l$ channels and the $4 d \rightarrow \varepsilon^{\prime} l^{\prime}$ channels, i.e.,

$$
\left\langle i|T| 5 p^{-1} \varepsilon l\right\rangle+\int d \varepsilon^{\prime} \sum_{l^{\prime}}\left\langle i|T| 4 d^{-1} \varepsilon^{\prime} l^{\prime}\right\rangle \frac{\left\langle 4 d^{-1} \varepsilon^{\prime} l^{\prime}|T| 5 p^{-1} \varepsilon l\right\rangle}{\Delta E}
$$

where $4 d^{-1}$ and $5 d^{-1}$ represent vacancies in the respective subshells. The second term in (26) dominates in the region where the $4 d$ cross section is large.

This result is of importance for a number of reasons. First, such phenomena are by no means limited to this particular case, but occur throughout the periodic table, for both ground and excited states [94], for almost all subshell cross sections in the vicinity of a new threshold. Second, measurement of the cross section, and the effect of interchannel coupling as exemplified by Figure 12, gives an experimental characterization of the strength of multichannel/interchannel effects. While they affect photoionization cross sections in other situations as well, in the type of experiment reported in Figure 12, multichannel interactions show a clear fingerprint. Finally, the analysis discussed in this section give us a clear a priori guide as to where multichannel effects are likely to be important: in situations where a weak channel is degenerate with a strong one.

\section{Concluding Remarks}

In this introductory paper on atomic photoionization with high-brightness light sources we have presented a large number of examples of phenomena whose study would be impossible without such sources. Not surprising, perhaps, is the fact that nearly all of these examples concern processes involving either $s$ subshells or satellite states (or both) as such processes have small cross sections. Nevertheless, much is gained by the study of these weak processes precisely because they are weak and therefore are susceptible to influences that have little effect on stronger transition processes.

Among the examples we have presented are many that exhibit our stated goal of attempting to extract simplicity from the complexity of detail often found in present-day measurements. Thus, measurements of doubly excited state spectra of $\mathrm{H}^{-}$and He have led to a significant amount of theoretical activity on three-body Coulomb states that has already resulted in new propensity rules for photoionization to these states (Section 3.1). Key features of satellite states have been shown to be amenable to interpretation based on single-configuration theoretical models (Sections 3.3.1, 3.5, and 3.6) and to measurement by means of fluorescence spectroscopy (Section 3.4). Also, satellite states have been demonstrated to have major influences on weak inner-shell photoionization channels near threshold (Section 3.2), as do strong photoionization channels at energies near their threshold (Section 3.8). Both fine-structure branching ratios (Section 3.3.2) and photoelectron angular distribution asymmetry parameters for satellite states (Section 3.6) have been shown to be interpretable using primarily angular momentum analyses. Finally, double photoionization processes are becoming much better understood, particularly in the asymptotic region (Section 3.7).

All of these examples show that photoionization studies of atoms with high-brightness light sources represent an ideal laboratory for uncovering fundamental new physics. This new physics includes further advances in descriptions of three-body Coulomb systems. It also includes the development of propensity rules and selection rules for processes which illustrate the effects of relativistic interactions and of electron correlations on complex many-body systems. 


\section{Acknowledgments}

A.F.S. gratefully acknowledges numerous discussions with James A. R. Samson, particularly concerning fluorescence spectroscopy. The work of A.F.S. reported herein was supported in part by the National Science Foundation under Grant Nos. PHY-8026055, PHY-8908605, and PHY-9108002. The work of S.T.M. reported herein was supported in part by the National Science Foundation under Grant No. PHY-9107539 and in part by the U.S. Army Research Office under Grant NO. DAAL03-89-K-0098.

\section{References}

1. D. R. Bates, Mon. Not Ry. Astron. Soc. 106, 432 (1946).

2. H. A. Bethe and E. E. Salpeter Quantum Mechanics of One- and Two-Electron Atoms (Springer, Berlin, 1957), pp. 247-323.

3. U. Fano and J. Cooper, Rev. Mod. Phys. 40, 441 (1968).

4. S. T. Manson Adv. Electron. Electron Phys. 41, 73 (1976).

5. S. T. Manson and D. Dill, In Electron Spectroscopy, Vol. 2, edited by C. R. Brundle and A. D. Baker (Academic Press, New York, 1978), pp. 157-195.

6. A. F. Starace. In Handbuch der Physik, Vol. 31, edited by W. Mehlhorn (Springer, Berlin, 1982), pp. 1-121.

7. M. Y. Amusia, Atomic Photoeffect (Plenum Press, New York, 1990).

8. C. N. Yang, Phys. Rev. 74, 764 (1948).

9. H. A. Bethe, In Handbuch der Physik, Vol. 24/1, edited by H. Geiger and K. Scheel (Springer, Berlin, 1933), p. 482.

10. J. Cooper and R. N. Zare, J. Chem. Phys. 48, 942 (1968).

11. S. T. Manson and A. F. Starace, Rev. Mod. Phys. 54, 389 (1982), and references therein.

12. D. Dill, Phys. Rev. A 7, 1976 (1973), and references therein.

13. U. Fano and D. Dill, Phys. Rev. A 6, 185 (1972).

14. P. G. Harris, H. C. Bryant, A. H. Mohagheghi, R. A. Reeder, H. Sharifian, C. Y. Tang, H. Tootoonchi, J. B. Donahue, C. R. Quick, D. C. Rislove, W. W. Smith, and J. E. Stewart Phys. Rev. Lett. 65, 309 (1990).

15. M. Domke, C. Xue, A. Puschmann, T. Mandel, E. Hudson, D. A. Shirley, G. Kaindl, C. H. Greene, H. R. Sadeghpour, and, H. Petersen, Phys. Rev. Lett. 66, 1306 (1991).

16. R. P. Madden and K. Codling, Astrophys. J. 141, 364 (1965).

17. J. W. Cooper, U. Fano, and F. Prats, Phys. Rev. Lett. 10, 518 (1963).

18. J. Macek, J. Phys. B 1, 831 (1968).

19. H. R. Sadeghpour and C. H. Greene, Phys. Rev. Lett. 65, 313 (1990).

20. X.-H. Liu, Z. Chen, and C. D. Lin, Phys. Rev. A 44, 5468 (1991).

21. J. M. Rost, J. S. Briggs, and J. M. Feagin, Phys. Rev. Lett. 66.1642 (1991).

22. J. M. Feagin and J. S. Briggs, Phys. Rev. A 37, 4599 (1988); J. M. Feagin, In Fundamental Processes of Atomic Dynamics, edited by J. S. Briggs, H. Kleinpoppen, and H. O. Lutz (Plenum Press, New York, 1988), pp. 275-300.

23. J. M. Rost and J. S. Briggs, J. Phys. B 23, L339 (1990).

24. H. R. Sadeghpour, C. H. Greene, and M. Cavagnero, Phys. Rev. A 45, 1587 (1992).

25. H. R Sadeghpour, Phys. Rev. A 43, 5821 (1991).

26. J. L. Dehmer and D. Dill, Phys. Rev. Lett. 37, 1049 (1976).

27. W. Ong and S. T. Manson, J. Phys. B 11, L65 (1978); Phys. Rev. A 19, 688 (1979).

28. W. R. Johnson and K. T. Cheng, Phys. Rev. Lett. 40, 1167 (1978); Phys. Rev. A 20, 978 (1979).

29. M. G. White, S. H. Southworth, P. Kobrin, E. D. Poliakoff, R. A. Rosenberg, and D. A. Shirley, Phys. Rev. Lett. 43, 1661 (1979).

30. K.-N. Huang and A. F. Starace, Phys. Rev. A 21, 697 (1980).

31. H. Derenbach and V. Schmidt, J. Phys. B 16, L337 (1983).

32. A. Fahlman, T. A. Carlson, and M. O. Krause, Phys. Rev. Lett. 50, 1114 (1983). 
33. G. Wendin and A. F. Starace, Phys. Rev. A 28, 3143 (1983).

34. See, e.g., G. Wendin, Struct. Bonding (Berlin) 45, 1 (1981), and references therein.

35. G. Wendin, Phys. Scr. 16, 296 (1977); see also G. Wendin and M. Ohno, Phys. Scr. 14, 148 (1976) for the analogous case of a $4 p$ hole state.

36. J. E. Hansen, J. Opt. Soc. Am. 67, 754 (1977); J. E. Hansen and D. W. Persson, Phys. Rev. A 18, 1459 (1978).

37. M. Y. Adam, F. Wuilleumier, N. Sandner, V. Schmidt, and D. G. Wendin, J. Phys. (Paris) 39, 129 (1978).

38. J. Tulkki, Phys. Rev. Lett. 62, 2817 (1989).

39. T. Gustafsson, Chem. Phys. Lett. 51, 383 (1977).

40. M. Y. Adam, thesis, L’Université de Paris-Sud, Centre d'Orsay, France (1978).

41. J. A. R. Samson and J. L. Gardner, Phys. Rev. Lett. 33, 671 (1974).

42. C. D. Caldwell, M. G. Flemming, M. O. Krause, P. Van Der Meulen, C. Pan, and A. F. Starace, Phys. Rev. A 41, 542 (1990).

43. J. M. Blatt and V. F. Weisskopf, Theoretical Nuclear Physics (Wiley, New York, 1952), Chap. viii, Sect. 3.

44. G. Howat, T. Åberg, and O. Goscinski, J. Phys. B 11, 1575 (1978).

45. D. Petrini (unpublished); see also J. Phys. B 14, 3839 (1981) for analogous calculations for B ${ }^{+}$.

46. See, e.g., J.-Z. Wu, S. B. Whitfield, C. D. Caldwell, M. O. Krause, P. van der Meulen, and A. Fahlman, Phys. Rev. A 42, 1350 (1990); C. D. Caldwell and M. O. Krause, J. Phys. B 23, 2233 (1990); M. G. Flemming, J- Z. Wu, C . D. Caldwell, and M. O. Krause, Phys. Rev. A 44, 1733 (1991); P. van der Meulen, M. O. Krause, and C. A. De Lange, J. Phys. B 25, 97 (1992).

47. W. C. Lineberger and B. W. Woodward, Phys. Rev. Lett. 25, 424 (1970).

48. H. Hotop, T. A. Patterson, and W. C. Lineberger, Phys. Rev. A 8, 762 (1973).

49. A. R. P. Rau and U. Fano, Phys. Rev. A 4, 1751 (1971).

50. A. R. P. Rau, In Electron and Photon Interactions with Atoms, edited by H. Kleinpoppen and M. R. C. McDowell (Plenum Press, New York, 1976), pp. 141-148.

51. P. A. Cox, Struct. Bonding 24, 59 (1975).

52. P. C. Engelking and W. C. Lineberger, Phys. Rev. A 19, 149 (1979).

53. J. Berkowitz and G. L. Goodman, J. Chem. Phys. 71, 1754 (1979).

54. J. Schirmer, L. S. Cederbaum, and J. Kiessling, Phys. Rev. A 22, 2696 (1980).

55. G. L. Goodman and J. Berkowitz, J. Chem. Phys. 94, 321 (1991).

56. C. Pan and A. F. Starace, Phys. Rev. A 47, 295 (1993).

57. M. O. Krause, C. D. Caldwell, S. B . Whiffleld, C. A. de Lange, and P. van der Meulen (unpublished).

58. F. Robicheaux and C. H. Greene, Phys. Rev. A 46, 3821 (1992).

59. F. Robicheaux and C. H. Greene (unpublished); F. Robicheaux (private communication).

60. See, e.g., the recent review by K. H. Schartner in, The Physics of Electronic and Atomic Collisions: XVI International Conference, New York, NY 1989. edited by A. Dalgarno, R. S. Freund, P. M. Koch, M. S. Lubell, and T. B. Lucatorto (AIP Conference Proceedings No. 205, New York, 1990), pp. 215-223.

61. P. R. Woodruff and J. A. R. Samson, Phys. Rev. Lett. 45, 110 (1980); Phys. Rev. A 25, 848 (1982).

62. K.-H. Schartner, B. Möbus, P. Lenz, H . Schmoranzer, and M. Wildberger, Phys. Rev. Lett. 61, 2744 (1988).

63. J. A. R. Samson, Y. Chung, and E. M. Lee, Phys. Rev. A 45, 259 (1992); J. A. R. Samson (private communication).

64. A. A. Wills, A. A. Cafolla, A. Svensson, and J. Comer, J. Phys. B 23, 2013 (1990).

65. D. Cubaynes, J. M. Bizau, F. J. Wuilleumier, B. Carré, and F. Gounand, Phys. Rev. Lett. 63, 2460 (1989).

66. B. I. Craig and F. P. Larkins, J. Phys. B. 18, 3569 (1985); 18, 3713 (1985).

67. Z. Felfli and S. T. Manson, Phys. Rev. Lett. 68, 1687 (1992).

68. M. Richter, J. M. Bizau, D. Cubaynes, T. Menzel, F. J. Wuilleumier, and B. Carré, Europhys. Lett. 12, 35 (1990).

69. D. Cubaynes, J. M . Bizau, M. Richter, and F. J. Wuilleumier, In VUV 10. Scientific Program and Abstracts (Maison de la Chemie, Paris, 1992), p. Mo71.

70. D. Cubaynes, J. M . Bizau, T. J. Morgan, B, Carré, and F. J. Wuilleumier, In VUV 10. Scientific Program and Abstracts (Maison de la Chemie, Paris, 1992), p. Tu79.

71. H. Stapelfeldt, D. G. Papaioannou, L. D. Noordam, and T. F. Gallagher, Phys. Rev. Lett. 67, 3223 (1991). 
72. M. Meyer, E. v. Raven, B. Sonntag, and J. E. Hansen, Phys. Rev. A 43, 177 (1991).

73. F. J. Wuilleumier, D. L, Ederer, and J. L. Picqué, Adv. At. Mol. Phys. 23, 197 (1988).

74. F. J. Wuilleumier, D. Cubaynes, and J. M. Bizau, In Atomic and Molecular Physics, edited by C. Cisneros, T. J. Morgan, and I. Alvarez (World Scientific, Singapore, 1991), pp. 474-495.

75. E. S. Chang and K. T. Taylor, J. Phys. B 11, L507 (1978).

76. T. A. Carlson, D. R. Mullins, C. E. Beall, B. W. Yates, J. W. Taylor, D. W. Lindle, B. P. Pullen, and F. A. Grimm, Phys. Rev. Lett. 60, 1382 (1988).

77. B. Langer, J. Viefhaus, O. Hemmers, A. Menzel, R. Wehlitz, and U. Becker, In ICAP XIII. Book of Abstracts (XIII ICAP, Munich, 1992), p. A41.

78. B. Langer, Anregungsenergieabhängigkeit von Photoelektronensatelliten unter Spezieller Berücksichtigung des Schwellbereiches, Ph.D. thesis, Tech. Univ. Berlin (1992).

79. M. Brauner, J. S. Briggs, and H. Klar, J. Phys. B 22, 2265 (1989), and references therein.

80. A. Dalgarno and H. R. Sadeghpour, Phys. Rev. A 46, R3591 (1992), and references therein.

81. J. C. Levin, D. W. Lindle, N. Keller, R. D. Miller, Y. Azuma, N. Berrah Mansour, H. G. Berry, and I. A. Sellin, Phys. Rev. Lett. 67, 968 (1991); J. C. Levin, I. A. Sellin, B. M. Johnson, D. W. Lindle, R. D. Miller, N. Berrah, Y. Azuma, H. G. Berry, and D.-H. Lee, Phys. Rev. A 47, R16 (1993).

82. D. Cubaynes, J. M. Bizau, M. Richter, and F. J. Wuilleumier, In VUV 10. Scientific Program and Abstracts (Maison de la Chemie, Paris, 1992), p. Mo80.

83. (A) J. A. R. Samson, Phys. Rev. Lett. 65, 2861 (1990); R. J. Bartlett, P. J. Walsh, Z. X. He, Y. Chung, E.-M. Lee, and J. A. R. Samson, Phys. Rev. A 46, 5574 (1992); J. A. R. Samson, R. J. Bartlett, and Z. X. He, Phys. Rev. 46, 7277 (1992). (B) J. A. R. Samson (private communication).

84. N. Berrah, F. Heiser, R. Wehlitz, J. Levin, S. B. Whitfield, J. Viefhaus, I. A. Sellin, and U. Becker, J. Phys. IV Colloq. 3(C6), 197 (1993).

85. C. Pan and H. P. Kelly, J. Phys. B. 28, 5001 (1995).

86. K. Hino, Phys. Rev. A 47, 4845 (1993); K. Hino, T. Ishihara, F. Shimizu, N. Toshima, and J. H. McGuire, Phys. Rev. A 48, 1271 (1993).

87. J. A. R. Samson, C. H. Greene, and R. J. Bartlett, Phys. Rev. Lett. 71, 201 (1993).

88. L. R. Andersson and J. Burgdorfer, Phys. Rev. Lett. 71, 50 (1993).

89. R. I. Hall, A. G. McConkey, L. Avaldi, K. Ellis, M. A. MacDonald, G. Dawber, and G. C . King, J. Phys. B 25, 1195 (1992).

90. R. Wehlitz, O. Hemmers, B. Langer, A. Menzel, and U. Becker, In XVIII ICPEAC, Abstracts of Papers, Vol. 1, edited by T. Andersen, B. Fastrup, F. Folkmann, and H. Knudsen (ICPEAC, Aarhus, Denmark, 1993), p. 7.

91. F. Maulbetsch and J. S. Briggs, J. Phys. B 26, 1679 (1993); 26, L647 (1993).

92. R. Dörner, C. L. Cocke, J. Feagin, T. Vogt, V. Mergel, H. Khemliche, S. Kravis, J. Ullrich, M. Unversagt, L. Spielberger, M. Damrau, O. Jagutzki, I. Ali, K. Ullman, M. Jung, E. P. Kanter, B. Sonntag, M. H. Prior, E. Rotenberg, J. Denliger, T. Warwick, S. T. Manson, and H. Schmidt-Böcking, Phys. Rev. Lett. (Submitted).

93. J. M. Bizau, D. Cubaynes, P. Gérard, and F. J. Wuilleumier, Phys. Rev. A 40, 3002 (1989).

94. J. M. Bizau, D. Cubaynes, P. Gérard, J. Wuilleumier, J. L. Picqué, D. L. Ederer, B. Carré, and G. Wendin, Phys. Rev. Lett. 57, 306 (1986). 